Prepared in cooperation with the City of Colorado Springs, Colorado

\title{
Estimated Probabilities and Volumes of Postwildfire Debris Flows-A Prewildfire Evaluation for the Pikes Peak Area, El Paso and Teller Counties, Colorado
}

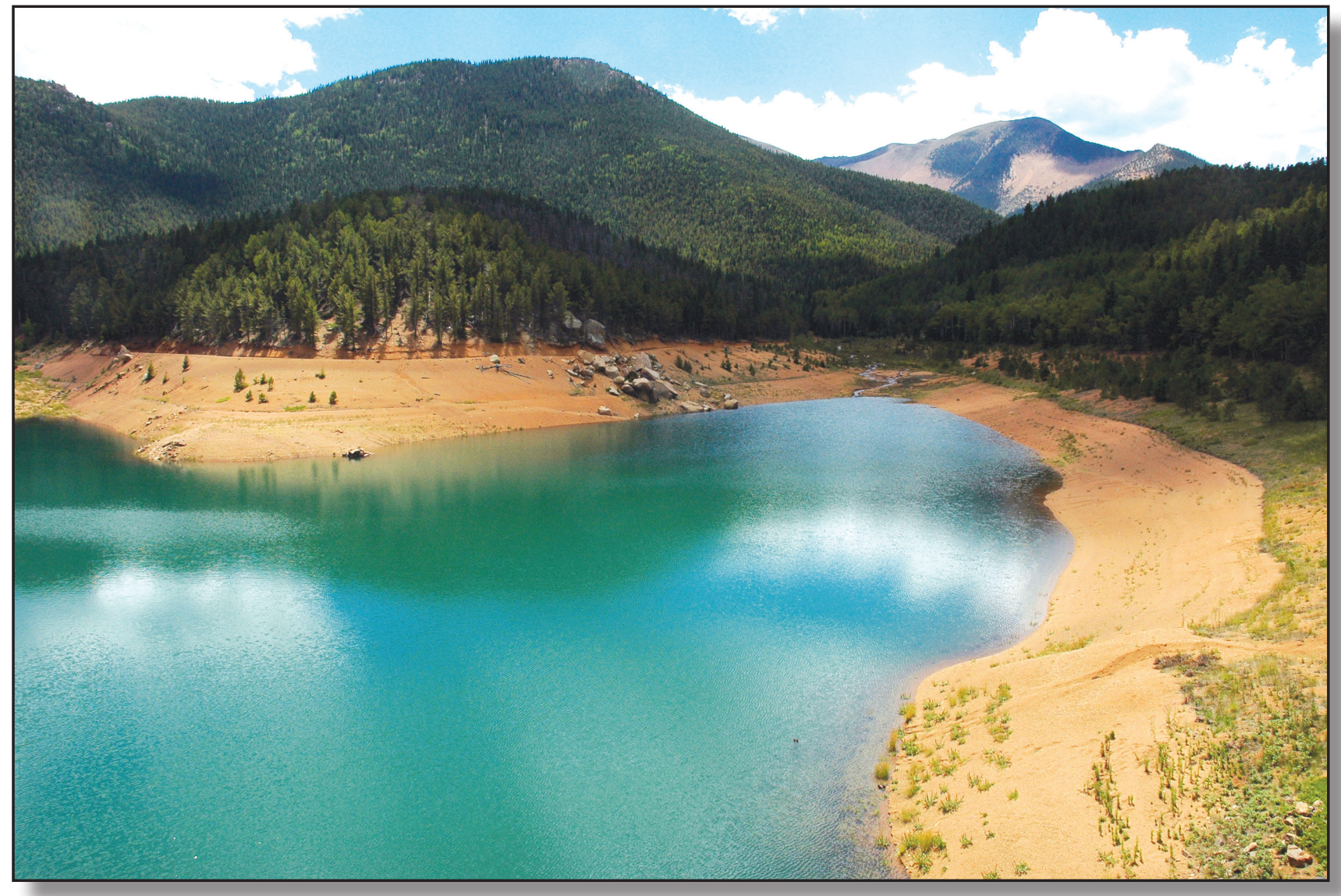

Scientific Investigations Report 2012-5104 
COVER: Big Tooth Reservoir. View is south-southwest to South Ruxton Creek and Almagre Mountain, elevation 12,367 feet, in Pike National Forest.

Photograph by John G. Elliott, U.S. Geological Survey, August 27, 2010. 


\section{Estimated Probabilities and Volumes of Postwildfire Debris Flows-A Prewildfire Evaluation for the Pikes Peak area, El Paso and Teller Counties, Colorado}

By John G. Elliott, Barbara C. Ruddy, Kristine L. Verdin, and Keelin R. Schaffrath

Prepared in cooperation with the City of Colorado Springs, Colorado

Scientific Investigations Report 2012-5104 


\title{
U.S. Department of the Interior \\ KEN SALAZAR, Secretary \\ U.S. Geological Survey \\ Marcia K. McNutt, Director
}

\author{
U.S. Geological Survey, Reston, Virginia: 2012
}

For more information on the USGS - the Federal source for science about the Earth, its natural and living resources, natural hazards, and the environment, visit http://www.usgs.gov or call 1-888-ASK-USGS.

For an overview of USGS information products, including maps, imagery, and publications, visit http://www.usgs.gov/pubprod

To order this and other USGS information products, visit http://store.usgs.gov

Any use of trade, product, or firm names is for descriptive purposes only and does not imply endorsement by the U.S. Government.

Although this report is in the public domain, permission must be secured from the individual copyright owners to reproduce any copyrighted materials contained within this report.

Suggested citation:

Elliott, J.G., Ruddy, B.C., Verdin, K.L., and Schaffrath, K.R., 2012, Estimated probabilities and volumes of postwildfire debris flows-A prewildfire evaluation for the Pikes Peak area, El Paso and Teller Counties, Colorado: U.S. Geological Survey Scientific Investigations Report 2012-5104, 26 p. 


\section{Contents}

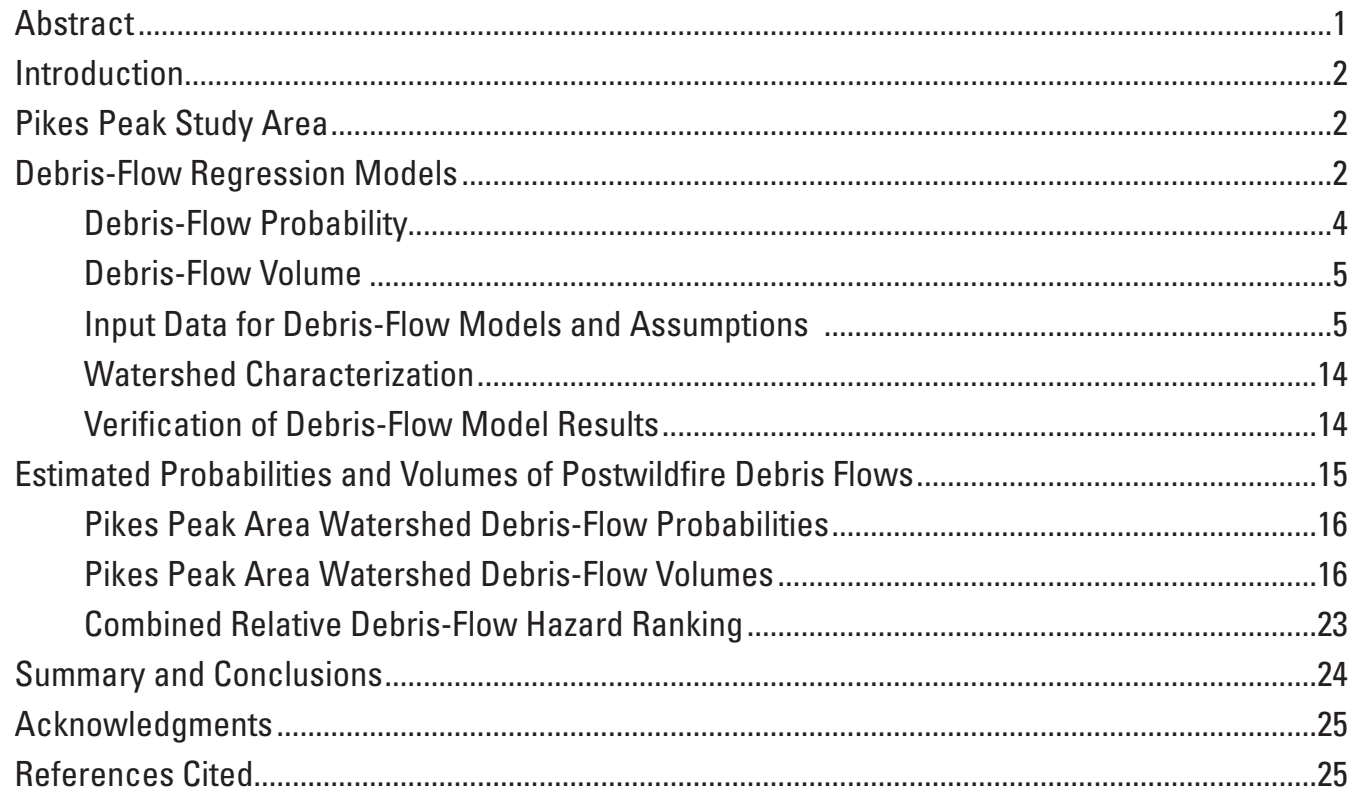

\section{Figures}

1. Shaded relief map of the Pikes Peak area showing topography, primary watersheds, and drainage networks.

2. Photograph of debris-flow marginal levee near the outlet of subwatershed $\mathrm{SCH} 13$, a tributary in the South Cheyenne Creek watershed

3. Photograph of debris-flow marginal levees bordering East Beaver Creek in subwatershed ROS08, a tributary that flows into the Penrose-Rosemont Reservoir .

4-9. Maps showing:

4. Primary watersheds and subwatersheds showing estimated debris-flow probabilities in response to the 2-year-recurrence, 1-hour-duration rainfall

5. Primary watersheds and subwatersheds showing estimated debris-flow probabilities in response to the 10 -year-recurrence, 1 -hour-duration rainfall .........18

6. Primary watersheds and subwatersheds showing estimated debris-flow probabilities in response to the 25-year-recurrence, 1-hour-duration rainfall .........19

7. Primary watersheds and subwatersheds showing estimated debris-flow volumes in response to the 2-year-recurrence, 1-hour-duration rainfall

8. Primary watersheds and subwatersheds showing estimated debris-flow volumes in response to the 10-year-recurrence, 1-hour-duration rainfall.

9. Primary watersheds and subwatersheds showing estimated debris-flow volumes in response to the 25 -year-recurrence, 1 -hour-duration rainfall

\section{Tables}

1. Infrastructure of concern to Colorado Springs Utilities and primary watersheds .4

2. Debris-flow model input variables and estimated debris-flow probabilities and volumes for primary watersheds and subwatersheds. 


\section{Conversion Factors}

Inch/Pound to SI

\begin{tabular}{|c|c|c|}
\hline Multiply & By & To obtain \\
\hline \multicolumn{3}{|c|}{ Length } \\
\hline inch (in.) & 25.4 & millimeter $(\mathrm{mm})$ \\
\hline foot $(\mathrm{ft})$ & 0.3048 & meter $(\mathrm{m})$ \\
\hline mile (mi) & 1.609 & kilometer $(\mathrm{km})$ \\
\hline \multicolumn{3}{|c|}{ Area } \\
\hline acre & 0.4047 & hectare (ha) \\
\hline square mile $\left(\mathrm{mi}^{2}\right)$ & 259.0 & hectare (ha) \\
\hline square mile $\left(\mathrm{mi}^{2}\right)$ & 2.590 & square kilometer $\left(\mathrm{km}^{2}\right)$ \\
\hline \multicolumn{3}{|c|}{ Volume } \\
\hline acre-foot (acre-ft) & 0.001233 & cubic hectometer $\left(\mathrm{hm}^{3}\right)$ \\
\hline \multicolumn{3}{|c|}{ Flow rate } \\
\hline cubic foot per second $\left(\mathrm{ft}^{3} / \mathrm{s}\right)$ & 0.02832 & cubic meter per second $\left(\mathrm{m}^{3} / \mathrm{s}\right)$ \\
\hline \multicolumn{3}{|c|}{ Mass } \\
\hline ton, short $(2,000 \mathrm{lb})$ & 0.9072 & megagram $(\mathrm{Mg})$ \\
\hline ton per year (ton/yr) & 0.9072 & megagram per year $(\mathrm{Mg} / \mathrm{yr})$ \\
\hline \multicolumn{3}{|c|}{ Pressure } \\
\hline pound per square foot $\left(\mathrm{lb} / \mathrm{ft}^{2}\right)$ & 47.88 & newton per square meter $\left(\mathrm{N} / \mathrm{m}^{2}\right)$ \\
\hline \multicolumn{3}{|c|}{ Density } \\
\hline pound per cubic foot $\left(\mathrm{lb} / \mathrm{ft}^{3}\right)$ & 0.01602 & gram per cubic centimeter $\left(\mathrm{g} / \mathrm{cm}^{3}\right)$ \\
\hline
\end{tabular}

SI to Inch/Pound

\begin{tabular}{|c|c|c|}
\hline Multiply & By & To obtain \\
\hline \multicolumn{3}{|c|}{ Length } \\
\hline millimeter $(\mathrm{mm})$ & 0.03937 & inch (in.) \\
\hline meter $(\mathrm{m})$ & 3.281 & foot $(\mathrm{ft})$ \\
\hline kilometer $(\mathrm{km})$ & 0.6214 & mile (mi) \\
\hline \multicolumn{3}{|c|}{ Area } \\
\hline hectare (ha) & 2.471 & acre \\
\hline hectare (ha) & 0.003861 & square mile $\left(\mathrm{mi}^{2}\right)$ \\
\hline square kilometer $\left(\mathrm{km}^{2}\right)$ & 0.3861 & square mile $\left(\mathrm{mi}^{2}\right)$ \\
\hline \multicolumn{3}{|c|}{ Volume } \\
\hline cubic hectometer $\left(\mathrm{hm}^{3}\right)$ & 810.7 & acre-foot (acre-ft) \\
\hline \multicolumn{3}{|c|}{ Flow rate } \\
\hline cubic meter per second $\left(\mathrm{m}^{3} / \mathrm{s}\right)$ & 35.31 & cubic foot per second $\left(\mathrm{ft}^{3} / \mathrm{s}\right)$ \\
\hline \multicolumn{3}{|c|}{ Mass } \\
\hline megagram $(\mathrm{Mg})$ & 1.102 & ton, short $(2,000 \mathrm{lb})$ \\
\hline megagram per year $(\mathrm{Mg} / \mathrm{yr})$ & 1.102 & ton per year (ton/yr) \\
\hline \multicolumn{3}{|c|}{ Pressure } \\
\hline newton per square meter $\left(\mathrm{N} / \mathrm{m}^{2}\right)$ & 0.02088 & pound per square foot $\left(\mathrm{lb} / \mathrm{ft}^{2}\right)$ \\
\hline \multicolumn{3}{|c|}{ Density } \\
\hline gram per cubic centimeter $\left(\mathrm{g} / \mathrm{cm}^{3}\right)$ & 62.4220 & pound per cubic foot $\left(\mathrm{lb} / \mathrm{ft}^{3}\right)$ \\
\hline
\end{tabular}

Vertical coordinate information is referenced to the North American Vertical Datum of 1988 (NAVD 88).

Horizontal coordinate information is referenced to the North American Datum of 1983 (NAD 83). 


\title{
Estimated Probabilities and Volumes of Postwildfire Debris Flows-A Prewildfire Evaluation for the Pikes Peak Area, El Paso and Teller Counties, Colorado
}

\author{
By John G. Elliott, Barbara C. Ruddy, Kristine L. Verdin, and Keelin R. Schaffrath
}

\section{Abstract}

Debris flows are fast-moving, high-density slurries of water, sediment, and debris that can have enormous destructive power. Although debris flows, triggered by intense rainfall or rapid snowmelt on steep hillsides covered with erodible material, are a common geomorphic process in some unburned areas, a wildfire can transform conditions in a watershed with no recent history of debris flows into conditions that pose a substantial hazard to residents, communities, infrastructure, aquatic habitats, and water supply. The location, extent, and severity of wildfire and the subsequent rainfall intensity and duration cannot be known in advance; however, hypothetical scenarios based on empirical debris-flow models are useful planning tools for conceptualizing potential postwildfire debris flows. A prewildfire study to determine the potential for postwildfire debris flows in the Pikes Peak area in El Paso and Teller Counties, Colorado, was initiated in 2010 by the U.S. Geological Survey, in cooperation with the City of Colorado Springs, Colorado Springs Utilities. The study was conducted to provide a relative measure of which subwatersheds might constitute the most serious potential debris-flow hazards in the event of a large-scale wildfire and subsequent rainfall.

Potential postwildfire debris-flow probabilities and volumes for 14 primary watersheds upstream from critical municipal-water infrastructure and 170 selected subwatersheds located within the primary watersheds were estimated by using empirical debris-flow models. The debris-flow models assumed that all of the forest and shrub cover in the watershed would burn at moderate- to high-burn severity. Three postwildfire precipitation scenarios were used to represent a range of likely precipitation that could occur within 4 to 6 years after a wildfire: (1) a 2-year recurrence, 1-hour duration rainfall, referred to as a 2-year storm; (2) a 10-year recurrence, 1-hour duration rainfall, referred to as a 10-year storm; and (3) a 25-year recurrence, 1-hour duration rainfall, referred to as a 25-year storm. Each of the precipitation scenarios indicated the possibility of debris flows from the hypothetically burned watersheds.
Estimated probabilities for postwildfire debris flows in the 170 subwatersheds range from less than 1 to 46 percent in response to the 2-year storm, from 1 to 67 percent in response to the 10 -year storm, and from 1 to 72 percent in response to the 25-year storm. Forty of the 170 subwatersheds have a greater than 60-percent probability of producing a debris flow in response to the 25-year storm. Subwatersheds with the lowest postwildfire debris-flow probabilities tend to have large areas of alpine and subalpine vegetation or other areas with sparse forest cover that would be minimally affected by wildfire. Subwatersheds with the highest debrisflow probabilities tend to have steep slopes and heavy forest cover. Postwildfire debris-flow probabilities for the 14 primary watersheds range from 4 to 42 percent in response to the 2-year storm, from 8 to 64 percent in response to the 10 -year storm, and from 10 to 70 percent in response to the 25-year storm.

Estimated volumes for postwildfire debris flows in the 170 subwatersheds range from less than $100 \mathrm{~m}^{3}$ to greater than $100,000 \mathrm{~m}^{3}$ in response to the 2-year storm, the 10 -year storm, and the 25-year storm. Estimated debris-flow volumes for each subwatershed increase as the storm recurrence interval increases. Subwatersheds with the smallest estimated postwildfire debris-flow volumes tend to have small drainage areas, have a small percent area of steep hillslopes, and (or) be located in alpine and subalpine zones. Subwatersheds with the largest estimated debris-flow volumes are those with the largest drainage areas. Estimated debris-flow volumes for the 14 primary watersheds range from about 11,000 to greater than $100,000 \mathrm{~m}^{3}$ in response to the 2-year storm, from about 14,000 to greater than $100,000 \mathrm{~m}^{3}$ in response to the 10 -year storm, and from about 15,000 to greater than $100,000 \mathrm{~m}^{3}$ in response to the 25 -year storm.

The subwatersheds associated with the greatest potential postwildfire and postprecipitation debris-flow hazards are those with a combination of a high probability of debrisflow occurrence and a large estimated volume of debris-flow material. The ten subwatersheds with the greatest combined relative debris-flow hazard rankings are in the watersheds of Cascade, South Ruxton, Ruxton, Gould, East Beaver, North Cheyenne, and South Cheyenne Creeks. 


\section{Introduction}

One of the most devastating potential postwildfire hazards is a debris flow (Cannon, 2001; Cannon and others, 1998). Debris flows are fast-moving, high-density slurries of water, sediment, and debris that can have enormous destructive power (Costa and Jarrett, 1981; Hungr and others, 1984; Pierson and Costa, 1987; Costa, 1988). Debris flows typically are triggered by intense rainfall or rapid snowmelt on steep hillsides covered with erodible material (Griffiths and others, 1996; Gartner and others, 2008). Although debris flows are a common geomorphic process in some unburned areas, a wildfire can transform conditions in a watershed with no recent history of debris flows into conditions that pose a substantial hazard to residents, communities, infrastructure, aquatic habitats, and water supply. Researchers have developed new techniques to estimate potential postwildfire debris-flow hazards (Cannon and others, 2010). These techniques can be used in a prewildfire analysis to estimate debris-flow hazards to life, property, infrastructure, and water resources before wildfires occur (Stevens and others, 2008; Elliott and others, 2011).

Several watersheds located to the north, east, and south of Pikes Peak are critical sources of municipal water for the cities of Colorado Springs and Manitou Springs, Colo. (fig. 1). Water collection systems located in these watersheds include reservoirs, tunnels, and diversion intake structures (table 1), which are all susceptible to damage or reduced operational efficiency from accelerated erosion and sedimentation that can occur following a wildfire.

Colorado experienced severe drought conditions in the late 20th and early 21 st centuries (Kuhn, 2005) which, when combined with the accumulation of forest fuel, can lead to increased wildfire activity. Widespread Colorado wildfires in 2002 were associated with a prolonged period of belowaverage spring and summer precipitation, high temperatures, and low humidity (Pielke and others, 2005). In 2010, the U.S. Geological Survey (USGS), in cooperation with the City of Colorado Springs, Colorado Springs Utilities (CSU), initiated a prewildfire study to determine the potential for postwildfire debris flows in 14 primary watersheds and 170 selected subwatersheds located within the primary watersheds with infrastructure of concern to CSU (table 1).

The objective of this study was to estimate the probability of postwildfire debris flows and to estimate the approximate volumes of debris flows that could be delivered from watersheds upstream from critical CSU infrastructure in order to provide a relative measure of which watersheds might constitute the most serious postwildfire debris-flow hazards. Although the location, percentage of burned area, severity of wildfire, and storm intensity and duration after a wildfire cannot be known in advance, hypothetical or design scenarios, such as those used in this report, are useful planning tools for conceptualizing potential postwildfire effects (Elliott and others, 2011). Flooding and other fluvial erosion processes that could cause substantial damage also can occur under postwildfire conditions, but were beyond the scope of this study.
This report provides estimates of probabilities and volumes of postwildfire debris-flows that could be produced within a few years after an assumed moderate- to high-severity wildfire. For each of the 14 primary watersheds (fig. 1) and 170 selected subwatersheds within these primary watersheds (table 1), it was assumed that the hypothetical wildfire would burn all forest- and shrub-covered areas. Using information provided in this report, CSU water-resource managers can plan prevention and mitigation strategies in advance of the occurrence of wildfires. Also, in the event of a large wildfire, this information will help managers identify the watersheds and subwatersheds with the greatest postwildfire debris-flow hazards (Ruddy and others, 2010).

\section{Pikes Peak Study Area}

The Pikes Peak study area encompasses 229 square kilometers $\left(\mathrm{km}^{2}\right)$ (88.4 square miles $\left.\left(\mathrm{mi}^{2}\right)\right)$ of rugged, mostly National Forest land in Teller and El Paso Counties (fig. 1). Elevation in the study area ranges from about 1,975 meters (m) (6,480 feet (ft)) at the outflow of the South Cheyenne Creek primary watershed (SCH00, table 1) to $4,301 \mathrm{~m}(14,110 \mathrm{ft})$ at the summit of Pikes Peak (U.S. Geological Survey, 2010). The majority of the study area is above 2,290 $\mathrm{m}$ (7,500 ft) elevation, except for small areas near the outflow of primary watersheds Ruxton Creek (MAN00, table 1), North Cheyenne Creek (NCH00, table 1), and South Cheyenne Creek (SCH00, table 1). The majority of the study area is composed of granite of Middle Proterozoic age with a few isolated areas of glacial drift of Quaternary age (Green, 1992). The study area is forested below an elevation of approximately $3,500 \mathrm{~m}(11,500 \mathrm{ft})$, and above this elevation, it is vegetated by alpine tundra.

Mean annual precipitation (1971-2000) varies throughout the study area and, when extrapolated to the area of the primary watersheds, ranges from about 570 millimeters $(\mathrm{mm})$ (22.4 inches (in.)) in the South Catamount Creek watershed (SCT00, table 1) to $775 \mathrm{~mm}$ (30.5 in.) in the Mason Reservoir portion of the Boehmer Creek watershed (MAS00, table 1) (U.S. Geological Survey, 2010). Much of the precipitation occurs in the summer as afternoon thunderstorms or as winter snow.

\section{Debris-Flow Regression Models}

Equations developed by Cannon and others (2010) were used to estimate the probability of debris-flow occurrence and estimate the volumes of debris flows that might occur, if fires of moderate to high severity consumed all forest- and shrub-covered areas in the 14 primary watersheds (figure 1 and table 1) and in the 170 selected subwatersheds within these larger primary watersheds. Primary watersheds and subwatersheds hereinafter are referred to collectively as watersheds. The probability and volume equations are based 


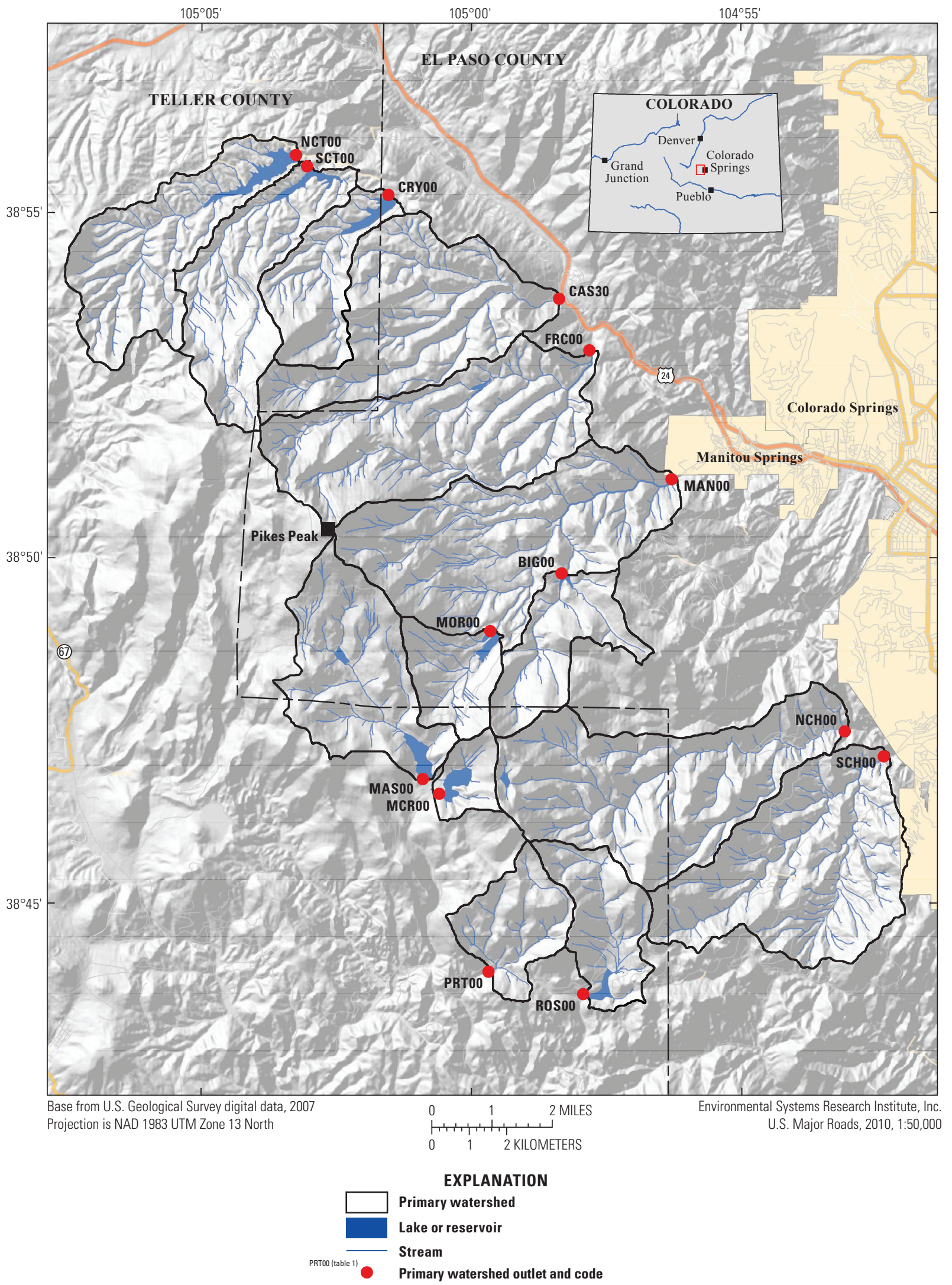

Figure 1. Shaded relief map of the Pikes Peak area showing topography, primary watersheds, and drainage networks. 
Table 1. Infrastructure of concern to Colorado Springs Utilities and primary watersheds.

[D M S, degrees, minutes, seconds; $\mathrm{km}^{2}$, square kilometers; $\mathrm{mi}^{2}$, square miles; No., number]

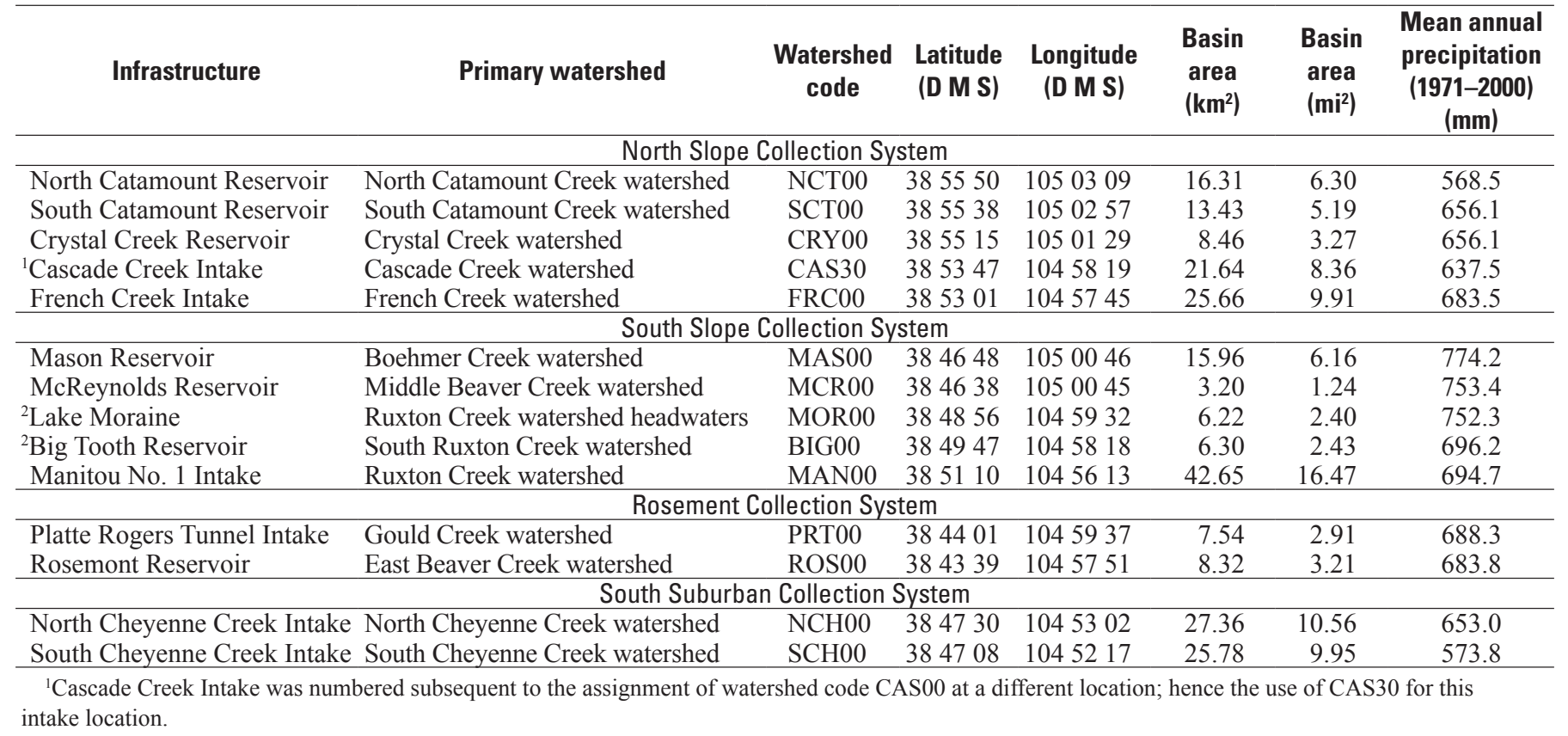

${ }^{2}$ Lake Moraine and Big Tooth Reservoir are located within the greater Ruxton Creek watershed and upstream from the Manitou No. 1 Intake.

on results from extensive studies of postwildfire debris flows that occurred in recently burned watersheds in the intermountain western United States, including Colorado, Utah, and California (Gartner and others, 2005; Gartner and others, 2008). The debris-flow equations are applicable only to areas where confined, channelized runoff is likely to occur (Cannon and others, 2010). The method of applying these equations to unburned watersheds to estimate the potential debris-flow hazards resulting from hypothetical wildfires was used by Elliott and others (2011) in the upper Blue River watershed in Summit County, Colorado.

The 14 primary watersheds in this study range in size from 3.20 to $42.65 \mathrm{~km}^{2}$ (1.24 to $16.47 \mathrm{mi}^{2}$ ), and the 170 subwatersheds range in size from 0.01 to $26.04 \mathrm{~km}^{2}$ ( 0.004 to $10.05 \mathrm{mi}^{2}$ ) (table 2). The watersheds examined by Cannon and others (2010) ranged in size from 0.01 to $103 \mathrm{~km}^{2}$; however, they found that postwildfire debris flows were not observed in watersheds with contributing drainage areas greater than approximately $30 \mathrm{~km}^{2}$. The 14 primary watersheds include the combined areas of multiple modeled subwatersheds within them as well as other areas within the primary watershed, such as large, laterally planar hillslopes, that were not modeled as a single landform. One primary watershed, Ruxton Creek (MAN00) has a drainage area that is greater than the maximum drainage area $\left(30 \mathrm{~km}^{2}\right)$ that produced a debris flow in Cannon and others (2010) because it includes the areas upstream from Lake Moraine (MOR00) and Big Tooth Reservoir (BIG00) (figure 1). Although greater in area than that observed by Cannon and others (2010), MAN00 is included in this analysis for the purpose of comparison.

\section{Debris-Flow Probability}

The regression equation of debris-flow probability is based on empirical data described by Cannon and others (2010, their model A). The equation is

$$
P=e^{x} /\left(1+e^{x}\right),
$$

where,

$P \quad$ is the probability of debris-flow occurrence in fractional form,

and

$$
\begin{aligned}
x & =-0.7+0.03(\% S G 30)-1.6(R)+0.06(\% A B) \\
& +0.07(I)+0.2(\% C)-0.4(L L),
\end{aligned}
$$

where,

$\% S G 30$ is the percentage of the watershed area with slopes equal to or greater than 30 percent;

$R \quad$ is watershed ruggedness, calculated as the change in watershed elevation (in meters) divided by the square root of the watershed area (in meters) (Melton, 1965);

$\% A B$ is the percentage of watershed area burned at moderate to high severity;

$I \quad$ is average storm intensity (in millimeters per hour);

$\% C$ is the clay content of the soil (in percent);

and

$L L \quad$ is the liquid limit of the soil (percentage of soil moisture by weight), which is the water content at which a soil changes from a plastic to a liquid state (Das, 1983). 
The debris-flow probability model of Cannon and others (2010) was developed using multiple logistic regression (Hosmer and Lemeshow, 2000) of data from postwildfire debris flows collected throughout the intermountain west. Logistic regression calculates McFadden's rho-squared, which is similar to the coefficient of determination, or $\mathrm{r}$-squared $\left(\mathrm{r}^{2}\right)$, of linear regression (SPSS, Inc., 2000), but rho-squared tends to be smaller than r-squared and also ranges from 0 to 1.0. Values of rho-squared between 0.20 and 0.40 indicate significant correlation (SPSS, Inc., 2000). McFadden's rho-squared calculated for this debris-flow probability model (Cannon and others, 2010, their model A) is 0.35 .

Cannon and others (2010) evaluated the sensitivity of their model as the number of watersheds known to have produced debris flows to the number of watersheds predicted by the model to have a probability of occurrence greater than 50 percent. The sensitivity of their model A, used in this report, was 44 percent (Cannon and others, 2010, their table 2).

\section{Debris-Flow Volume}

The multivariate regression equation for debris-flow volume developed by Cannon and others (2010, their equation 2) was used to estimate a mean volume of debrisflow material deposited at the outlet of a recently burned watershed in the upper Blue River watershed (Elliott and others, 2011), and was used in this study. The equation is

$\ln V=7.2+0.6(\ln S G 30)+0.7(A B)^{0.5}+0.2(T)^{0.5}+0.3$, where,

In is the natural logarithm;

$V$ is the debris-flow volume (including water, sediment, and debris) in cubic meters;

$S G 30$ is the area of watershed with slopes equal to or greater than 30 percent (in square kilometers);

$A B$ is the watershed area burned at moderate to high severity (in square kilometers);

$T$ is the total storm rainfall (in millimeters);

and

0.3 is a bias correction that changes the predicted estimate from a median to a mean value (Helsel and Hirsch, 2002).

The debris-flow volume equation has an $\mathrm{r}^{2}$ of 0.83 and a standard error of 0.90 . In model validation, the volume equation predicted 87 percent of the debris-flow volumes within the 95-percent prediction interval; all reported volumes were within one order of magnitude of predicted volumes (Cannon and others, 2010).

\section{Input Data for Debris-Flow Models and Assumptions}

Input data for postwildfire debris-flow probability and volume estimates in the Pikes Peak area were obtained from a variety of sources. The primary input variables of the debris-flow models used in this study, developed by Cannon and others (2010), are the extent of the burned area, rainfall volumes and intensity, and soil and topographic characteristics.

Forested area was used as a surrogate for the extent of burned area, and it was assumed that all of the forest and shrub cover, which was defined from the 1992 Enhanced National Land Cover Database (Nakagaki and others, 2007), would burn at moderate- to high-burn severity. Although this assumption may characterize only extensive and severe wildfires, it provides a consistent basis for comparison of debris-flow hazards among watersheds in the Pikes Peak area as well as providing a worst-case scenario for debris-flow prediction.

High-burn severity is defined by Lindsey (2002) as the complete consumption of the forest litter and duff and the combustion of all fine fuels in the canopy. A deep ash layer may be present on the forest floor in areas of high-burn severity, and the top layer of the mineral soil may be changed in color due to substantial soil heating where large-diameter fuels were consumed. Moderate-burn severity is defined as the consumption of forest litter and duff in discontinuous patches. Leaves or needles, although scorched, may remain on trees. Foliage and twigs on the forest floor are consumed, and some heating of the mineral soils may occur if the soil organic layer is thin.

Rainfall, in terms of both storm recurrence and precipitation duration, is an essential element in the generation of postwildfire debris flows. The debris flows studied by Cannon and others (2010) to develop equations 1 and 2 were generated by short-duration (up to 1-hour) convective rainstorms with recurrence intervals ranging from less than 2 years to as many as 10 years. Another researcher noted that the 25 -year recurrence rainfall might be more representative of storms that generate other debris flows because a more frequently occurring storm might deliver too little rainfall runoff to sustain a debris flow, whereas a less frequently occurring storm might deliver too much rainfall runoff, creating a sediment-laden water flood rather than a debris flow (J.S. O’Brien, FLO Engineering, Inc., oral commun., 2002).

Postwildfire studies of the 2002 Hayman, Coal Seam, and Missionary Ridge burned areas estimated that burned watersheds were the most vulnerable to extensive erosion and potential debris flows for a 4- to 6-year period following those wildfires (Elliott and others, 2005), whereas Cannon and others (2010) found that most postwildfire debris-flow activity occurred within about 2 years after the wildfire. Therefore, a 2-year recurrence rainfall is likely to occur while the burned area is most vulnerable to erosion, but such a storm might not represent a worst-case scenario. To represent weather conditions that might result in more severe postwildfire erosion, debris-flow probabilities and volumes in response to the 10-year and 25-year recurrence rainfall events also were simulated for the Pikes Peak area watersheds. 
Table 2. Debris-flow model input variables and estimated debris-flow probabilities and volumes for primary watersheds and subwatersheds.

[D M S, degrees, minutes, seconds; RI, recurrence interval: $\mathrm{km}^{2}$, square kilometer; $\mathrm{m} / \mathrm{m}$, meter per meter; $\mathrm{m}^{3}$, cubic meter; mm, millimeter; na, not applicable; $<$, less than; $>$, greater than]

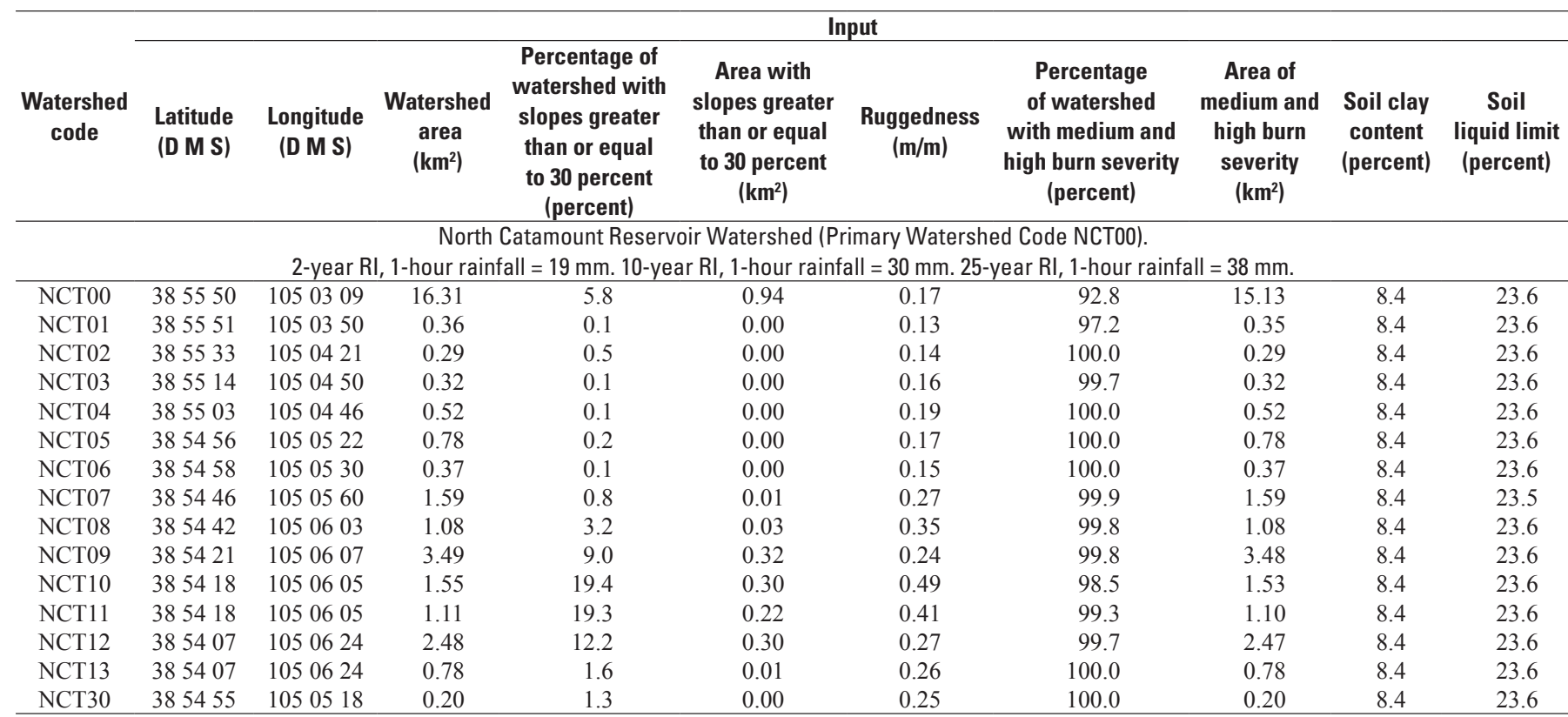

South Catamount Reservoir Watershed (Primary Watershed Code SCT00).

2-year $\mathrm{RI}, 1$-hour rainfall = $22 \mathrm{~mm}$. 10-year $\mathrm{RI}$, 1-hour rainfall = $34 \mathrm{~mm}$. 25-year RI, 1-hour rainfall $=39 \mathrm{~mm}$.

\begin{tabular}{|c|c|c|c|c|c|c|c|c|c|c|}
\hline SCT00 & 385538 & 1050257 & 13.43 & 8.7 & 1.17 & 0.32 & 82.3 & 11.05 & 10.0 & 24.8 \\
\hline SCT01 & 385508 & 1050322 & 1.34 & 2.0 & 0.03 & 0.20 & 99.2 & 1.33 & 8.4 & 23.6 \\
\hline SCT02 & 385509 & 1050345 & 0.49 & 3.1 & 0.02 & 0.32 & 99.9 & 0.49 & 8.4 & 23.6 \\
\hline SCT03 & 385507 & 1050350 & 10.74 & 10.5 & 1.12 & 0.36 & 79.6 & 8.54 & 10.4 & 25.1 \\
\hline SCT04 & 385425 & 1050441 & 3.59 & 16.6 & 0.60 & 0.57 & 60.5 & 2.17 & 11.1 & 25.7 \\
\hline SCT05 & 385346 & 1050432 & 2.44 & 23.4 & 0.57 & 0.62 & 42.1 & 1.03 & 12.3 & 26.6 \\
\hline SCT06 & 385416 & 1050455 & 2.78 & 13.5 & 0.38 & 0.54 & 91.0 & 2.53 & 9.8 & 24.7 \\
\hline SCT07 & 385344 & 1050509 & 1.72 & 21.7 & 0.37 & 0.61 & 85.5 & 1.47 & 10.6 & 25.3 \\
\hline SCT30 & 385508 & 1050346 & 10.77 & 10.4 & 1.12 & 0.36 & 79.7 & 8.58 & 10.4 & 25.1 \\
\hline
\end{tabular}

Crysyal Creek Reservoir Watershed (Primary Watershed Code CRY00).

\begin{tabular}{|c|c|c|c|c|c|c|c|c|c|c|}
\hline CRY00 & 385515 & 1050129 & 8.46 & 6.9 & 0.59 & 0.27 & 96.1 & 8.12 & 8.7 & 23.8 \\
\hline CRY01 & 385432 & 1050243 & 0.64 & 7.0 & 0.04 & 0.32 & 99.8 & 0.64 & 8.4 & 23.6 \\
\hline CRY02 & 385417 & 1050247 & 5.00 & 10.2 & 0.51 & 0.34 & 96.7 & 4.83 & 8.9 & 24.0 \\
\hline CRY03 & 385411 & 1050245 & 0.01 & 0.0 & 0.00 & 0.54 & 100.0 & 0.01 & 8.4 & 23.6 \\
\hline CRY04 & 385318 & 1050230 & 0.61 & 12.0 & 0.07 & 0.45 & 98.8 & 0.60 & 8.4 & 23.6 \\
\hline CRY05 & 385319 & 1050230 & 1.20 & 12.6 & 0.15 & 0.49 & 89.5 & 1.07 & 10.2 & 25.0 \\
\hline CRY30 & 385433 & 1050242 & 6.25 & 9.1 & 0.57 & 0.31 & 97.3 & 6.08 & 8.8 & 23.9 \\
\hline
\end{tabular}

Cascade Creek Watershed (Primary Watershed Code CAS30).

2-year RI, 1-hour rainfall = $26 \mathrm{~mm}$. 10-year RI, 1-hour rainfall $=38 \mathrm{~mm}$. 25-year RI, 1-hour rainfall $=44 \mathrm{~mm}$.

\begin{tabular}{|c|c|c|c|c|c|c|c|c|c|c|}
\hline CAS30 & 385347 & 1045819 & 21.64 & 13.8 & 2.99 & 0.37 & 95.2 & 20.60 & 8.9 & 24.0 \\
\hline CASO0 & 385349 & 1045911 & 20.10 & 11.0 & 2.21 & 0.32 & 94.9 & 19.08 & 8.9 & 24.0 \\
\hline CAS01 & 385346 & 1045908 & 0.01 & 24.6 & 0.00 & 1.49 & 100.0 & 0.01 & 8.4 & 23.6 \\
\hline CAS02 & 385351 & 1045915 & 9.04 & 13.6 & 1.23 & 0.47 & 89.7 & 8.11 & 9.5 & 24.4 \\
\hline CAS03 & 385344 & 1045941 & 1.01 & 15.6 & 0.16 & 0.28 & 99.6 & 1.01 & 8.4 & 23.6 \\
\hline CAS04 & 385401 & 1045926 & 10.83 & 8.1 & 0.88 & 0.21 & 99.1 & 10.74 & 8.4 & 23.6 \\
\hline CAS05 & 385401 & 1045949 & 4.97 & 6.3 & 0.31 & 0.26 & 99.2 & 4.93 & 8.4 & 23.6 \\
\hline CAS06 & 385321 & 1050005 & 1.50 & 4.7 & 0.07 & 0.44 & 99.7 & 1.49 & 8.4 & 23.6 \\
\hline CAS07 & 385322 & 1050006 & 5.61 & 15.3 & 0.86 & 0.56 & 83.6 & 4.69 & 10.1 & 24.9 \\
\hline CAS08 & 385350 & 1050014 & 1.05 & 5.3 & 0.06 & 0.39 & 98.5 & 1.04 & 8.4 & 23.6 \\
\hline CAS09 & 385329 & 1050034 & 0.82 & 4.6 & 0.04 & 0.44 & 99.6 & 0.82 & 8.4 & 23.6 \\
\hline CAS10 & 385320 & 1050048 & 2.18 & 16.9 & 0.37 & 0.38 & 98.9 & 2.16 & 8.4 & 23.6 \\
\hline CAS11 & 385312 & 1050123 & 0.24 & 25.9 & 0.06 & 0.84 & 99.6 & 0.24 & 8.4 & 23.6 \\
\hline CAS12 & 385313 & 1050133 & 1.24 & 23.0 & 0.29 & 0.45 & 98.5 & 1.22 & 8.4 & 23.6 \\
\hline CAS13 & 385412 & 1050047 & 2.68 & 6.0 & 0.16 & 0.31 & 99.8 & 2.68 & 8.4 & 23.6 \\
\hline CAS14 & 385413 & 1050046 & 1.39 & 2.0 & 0.03 & 0.18 & 99.6 & 1.39 & 8.4 & 23.6 \\
\hline CAS15 & 385416 & 1050060 & 0.57 & 5.6 & 0.03 & 0.42 & 99.8 & 0.57 & 8.4 & 23.6 \\
\hline CAS16 & 385235 & 1050222 & 2.53 & 16.3 & 0.41 & 0.55 & 66.1 & 1.67 & 12.2 & 26.5 \\
\hline CAS31 & 385350 & 1050012 & 1.05 & 5.3 & 0.06 & 0.39 & 98.5 & 1.04 & 8.4 & 23.6 \\
\hline
\end{tabular}


Table 2. Debris-flow model input variables and estimated debris-flow probabilities and volumes for primary watersheds and subwatersheds.-Continued

[DMS, degrees, minutes, seconds; RI, recurrence interval: $\mathrm{km}^{2}$, square kilometer; $\mathrm{m} / \mathrm{m}$, meter per meter; $\mathrm{m}^{3}$, cubic meter; mm, millimeter; na, not applicable; $<$, less than; >, greater than]

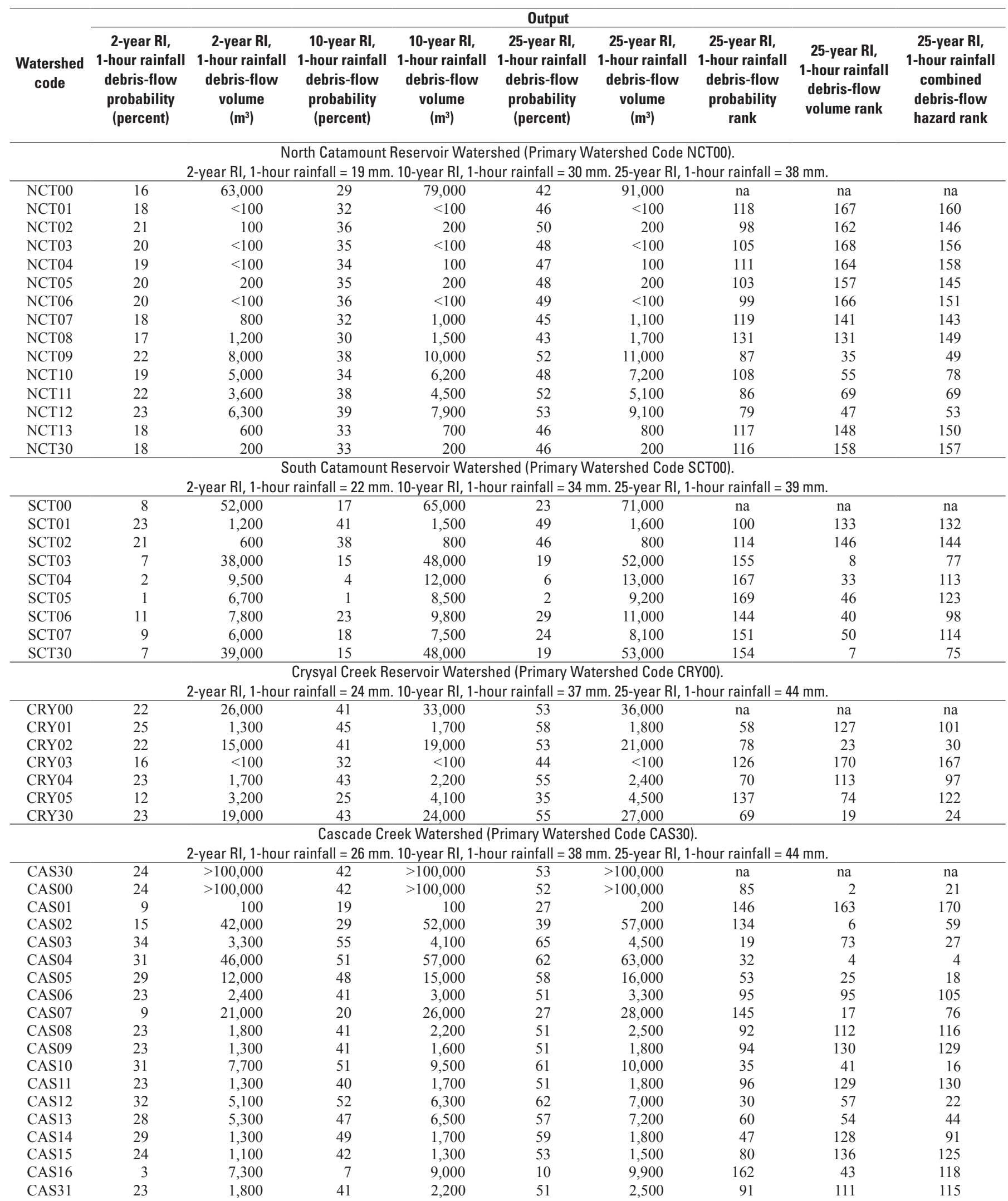


Table 2. Debris-flow model input variables and estimated debris-flow probabilities and volumes for primary watersheds and subwatersheds.-Continued

[D M S, degrees, minutes, seconds; RI, recurrence interval: $\mathrm{km}^{2}$, square kilometer; $\mathrm{m} / \mathrm{m}$, meter per meter; $\mathrm{m}^{3}$, cubic meter; mm, millimeter; na, not applicable; $<$, less than; $>$, greater than]

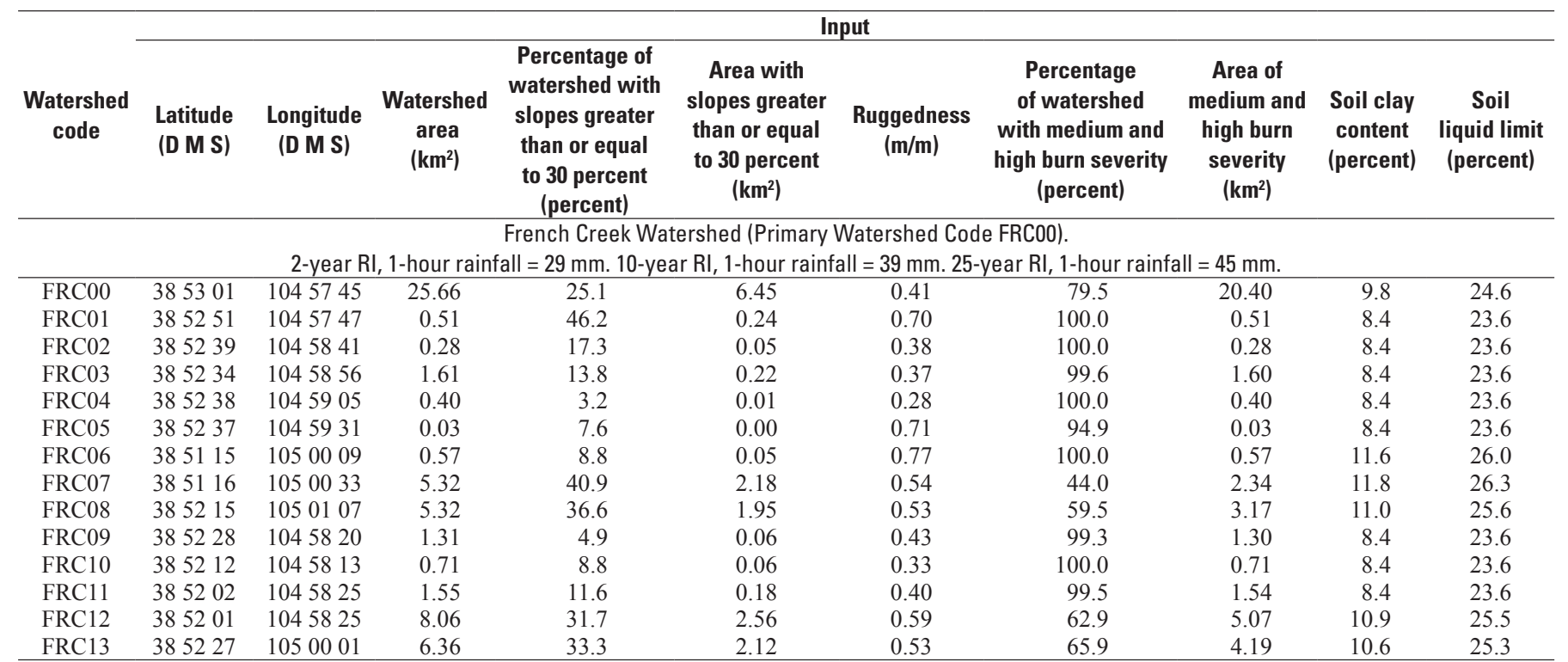

Mason Reservoir (Middle Beaver Creek) Watershed (Primary Watershed Code MAS00).

2-year $\mathrm{Rl}$, 1-hour rainfall $=30 \mathrm{~mm}$. 10-year $\mathrm{RI}$, 1-hour rainfall $=42 \mathrm{~mm}$. 25-year $\mathrm{RI}, 1$-hour rainfall $=44 \mathrm{~mm}$.

\begin{tabular}{|c|c|c|c|c|c|c|c|c|c|c|}
\hline MAS00 & 384648 & 1050046 & 15.96 & 4.5 & 0.71 & 0.24 & 62.2 & 9.92 & 11.6 & 26.0 \\
\hline MAS01 & 384716 & 1050048 & 0.51 & 2.6 & 0.01 & 0.30 & 95.8 & 0.49 & 8.4 & 23.6 \\
\hline MAS02 & 384735 & 1050123 & 1.75 & 2.2 & 0.04 & 0.37 & 80.8 & 1.41 & 9.8 & 24.7 \\
\hline MAS03 & 384738 & 1050142 & 2.49 & 1.7 & 0.04 & 0.26 & 84.0 & 2.09 & 12.6 & 26.9 \\
\hline MAS04 & 384813 & 1050206 & 1.64 & 5.2 & 0.08 & 0.28 & 60.1 & 0.99 & 12.8 & 27.0 \\
\hline MAS05 & 384838 & 1050233 & 2.74 & 3.3 & 0.09 & 0.51 & 31.7 & 0.87 & 12.8 & 27.0 \\
\hline MAS30 & 384732 & 1050122 & 0.08 & 7.0 & 0.01 & 0.62 & 100.0 & 0.08 & 9.0 & 24.1 \\
\hline
\end{tabular}

McReynolds Reservoir (Middle Beaver Creek) Watershed (Primary Watershed Code MCR00).

2-year $\mathrm{Rl}$, 1-hour rainfall $=30 \mathrm{~mm}$. 10-year $\mathrm{Rl}$, 1-hour rainfall $=42 \mathrm{~mm}$. 25-year $\mathrm{Rl}$, 1-hour rainfall $=46 \mathrm{~mm}$.

\begin{tabular}{lrlrrrrrrr}
\hline MCR00 & 384638 & 1050045 & 3.20 & 19.8 & 0.63 & 0.22 & 64.7 & 2.07 & 8.4 \\
MCR01 & 384633 & 1050014 & 0.45 & 32.4 & 0.15 & 0.65 & 68.1 & 0.31 & 8.4 \\
MCR30 & 384627 & 1050021 & 0.14 & 2.4 & 0.00 & 0.38 & 85.6 & 0.12 & 8.4 \\
MCR31 & 384658 & 1050007 & 0.49 & 19.8 & 0.10 & 0.63 & 55.1 & 23.6 \\
\hline
\end{tabular}

'Lake Moraine (Ruxton Creek) Watershed (Primary Watershed Code MOR00).

2-year Rl, 1-hour rainfall = $29 \mathrm{~mm}$. 10-year RI, 1-hour rainfall = $42 \mathrm{~mm}$. 25-year Rl, 1-hour rainfall = $46 \mathrm{~mm}$.

\begin{tabular}{|c|c|c|c|c|c|c|c|c|c|c|}
\hline MOR00 & 384856 & 1045932 & 6.22 & 17.4 & 1.08 & 0.28 & 86.2 & 5.36 & 8.8 & 23.9 \\
\hline MOR01 & 384833 & 1050000 & 4.55 & 20.6 & 0.94 & 0.33 & 89.3 & 4.07 & 8.7 & 23.8 \\
\hline MOR03 & 384848 & 1045947 & 0.73 & 2.7 & 0.02 & 0.77 & 89.0 & 0.65 & 9.4 & 24.4 \\
\hline
\end{tabular}

'Big Tooth Reservoir (South Ruxton Creek) Watershed (Primary Watershed Code BIG00).

2-year $\mathrm{Rl}$, 1-hour rainfall = $29 \mathrm{~mm}$. 10-year $\mathrm{RI}$, 1-hour rainfall $=42 \mathrm{~mm}$. 25-year RI, 1-hour rainfall $=46 \mathrm{~mm}$.

\begin{tabular}{|c|c|c|c|c|c|c|c|c|c|c|}
\hline BIG00 & 384947 & 1045818 & 6.30 & 15.6 & 0.98 & 0.36 & 85.1 & 5.36 & 8.4 & 23.6 \\
\hline BIG01 & 384938 & 1045811 & 0.49 & 5.9 & 0.03 & 0.57 & 97.7 & 0.48 & 8.4 & 23.6 \\
\hline BIG02 & 384938 & 1045812 & 2.08 & 16.1 & 0.33 & 0.33 & 99.9 & 2.08 & 8.4 & 23.6 \\
\hline BIG03 & 384935 & 1045823 & 6.06 & 16.2 & 0.98 & 0.37 & 84.9 & 5.14 & 8.4 & 23.6 \\
\hline BIG04 & 384923 & 1045822 & 0.62 & 26.6 & 0.16 & 0.55 & 100.0 & 0.62 & 8.4 & 23.6 \\
\hline BIG05 & 384842 & 1045847 & 1.21 & 9.9 & 0.12 & 0.50 & 93.4 & 1.13 & 8.4 & 23.6 \\
\hline BIG06 & 384841 & 1045847 & 2.66 & 21.6 & 0.57 & 0.47 & 69.7 & 1.86 & 8.4 & 23.6 \\
\hline
\end{tabular}

${ }^{2}$ Manitou No. 1 Intake (Ruxton Creek) Watershed (Primary Watershed Code MAN00).

2-year RI, 1-hour rainfall $=29 \mathrm{~mm}$. 10-year RI, 1-hour rainfall $=42 \mathrm{~mm}$. 25-year Rl, 1-hour rainfall $=46 \mathrm{~mm}$.

\begin{tabular}{|c|c|c|c|c|c|c|c|c|c|c|}
\hline MAN00 & 385110 & 1045613 & 42.65 & 20.4 & 8.70 & 0.34 & 85.3 & 36.38 & 9.7 & 24.5 \\
\hline MAN01 & 385101 & 1045632 & 0.94 & 40.8 & 0.38 & 1.00 & 99.0 & 0.93 & 8.8 & 23.8 \\
\hline MAN02 & 385105 & 1045638 & 0.24 & 28.9 & 0.07 & 1.10 & 99.0 & 0.24 & 20.8 & 30.8 \\
\hline MAN03 & 385106 & 1045655 & 1.48 & 19.3 & 0.28 & 0.43 & 100.0 & 1.48 & 10.5 & 24.8 \\
\hline MAN04 & 385058 & 1045709 & 1.15 & 57.9 & 0.67 & 0.78 & 95.2 & 1.09 & 8.4 & 23.6 \\
\hline MAN05 & 385057 & 1045726 & 0.49 & 28.9 & 0.14 & 0.72 & 96.2 & 0.47 & 8.4 & 23.6 \\
\hline MAN06 & 385054 & 1045743 & 0.30 & 20.4 & 0.06 & 0.54 & 98.6 & 0.29 & 8.4 & 23.6 \\
\hline MAN07 & 385047 & 1045810 & 5.08 & 12.6 & 0.64 & 0.73 & 72.8 & 3.70 & 10.6 & 25.3 \\
\hline
\end{tabular}


Table 2. Debris-flow model input variables and estimated debris-flow probabilities and volumes for primary watersheds and subwatersheds.-Continued

[DMS, degrees, minutes, seconds; RI, recurrence interval: $\mathrm{km}^{2}$, square kilometer; $\mathrm{m} / \mathrm{m}$, meter per meter; $\mathrm{m}^{3}$, cubic meter; mm, millimeter; na, not applicable; $<$, less than; >, greater than]

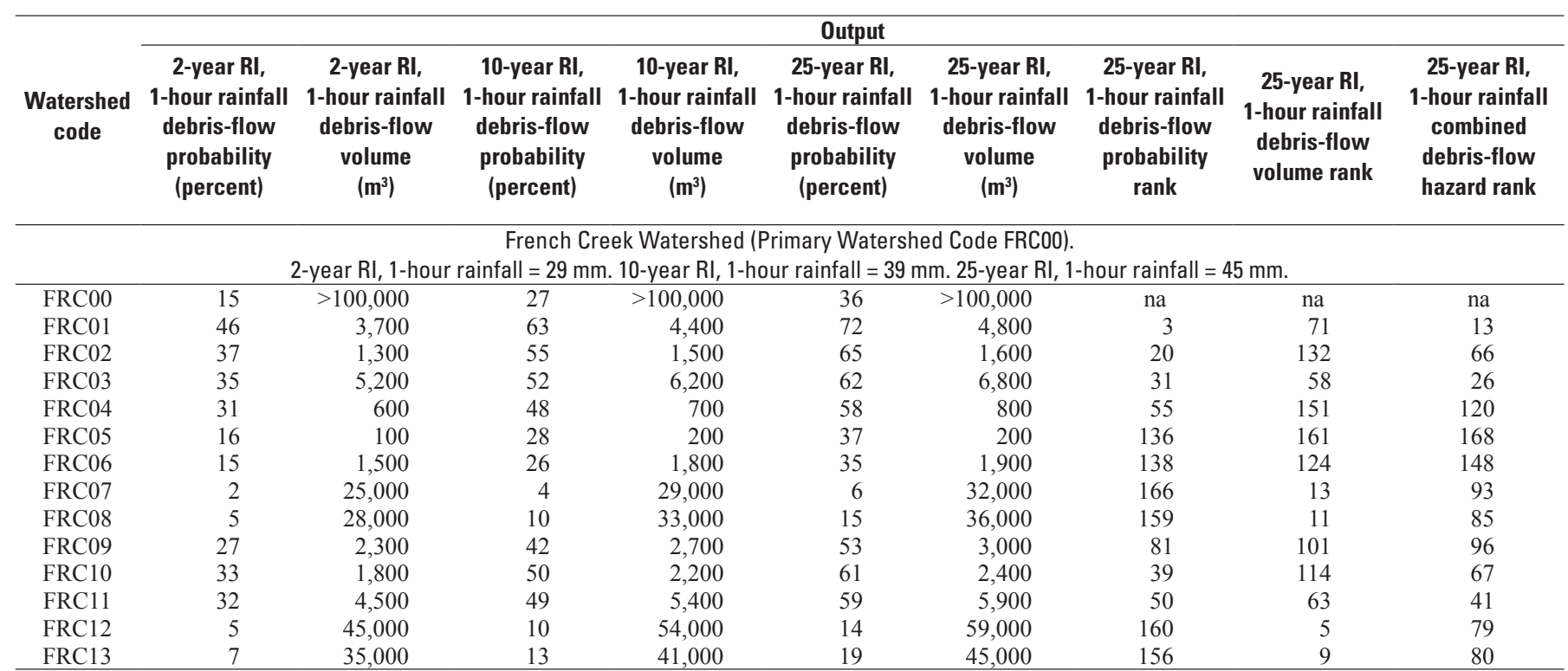

Mason Reservoir (Middle Beaver Creek) Watershed (Primary Watershed Code MAS00).

2-year $\mathrm{RI}$, 1-hour rainfall $=30 \mathrm{~mm}$. 10-year $\mathrm{RI}$, 1-hour rainfall $=42 \mathrm{~mm}$. 25-year RI, 1-hour rainfall $=44 \mathrm{~mm}$.

\begin{tabular}{|c|c|c|c|c|c|c|c|c|c|}
\hline MAS00 & 4 & 40,000 & 8 & 49,000 & 10 & 51,000 & na & na & na \\
\hline MAS01 & 27 & 700 & 46 & 800 & 49 & 800 & 101 & 147 & 139 \\
\hline MAS02 & 10 & 1,800 & 21 & 2,200 & 23 & 2,200 & 152 & 116 & 153 \\
\hline MAS03 & 11 & 2,200 & 21 & 2,700 & 24 & 2,800 & 148 & 104 & 141 \\
\hline MAS04 & 3 & 2,500 & 6 & 3,000 & 7 & 3,100 & 164 & 97 & 147 \\
\hline MAS05 & $<1$ & 2,500 & 1 & 3,000 & 1 & 3,100 & 170 & 98 & 152 \\
\hline MAS30 & 23 & 300 & 41 & 400 & 44 & 400 & 125 & 153 & 159 \\
\hline \multicolumn{10}{|c|}{$\begin{array}{l}\text { McReynolds Reservoir (Middle Beaver Creek) Watershed (Primary Watershed Code MCR00). } \\
\text { 2-year RI, 1-hour rainfall = } 30 \mathrm{~mm} .10 \text {-year RI, 1-hour rainfall = } 42 \mathrm{~mm} .25 \text {-year RI, 1-hour rainfall = } 46 \mathrm{~mm} .\end{array}$} \\
\hline MCR00 & 10 & 11,000 & 20 & 14,000 & 25 & 15,000 & na & na & na \\
\hline MCR01 & 9 & 2,500 & 18 & 3,100 & 23 & 3,300 & 153 & 94 & 138 \\
\hline MCR30 & 15 & 200 & 29 & 300 & 35 & 300 & 140 & 155 & 166 \\
\hline MCR31 & 3 & 1,900 & 7 & 2,400 & 9 & 2,500 & 163 & 110 & 154 \\
\hline
\end{tabular}

'Lake Moraine (Ruxton Creek) Watershed (Primary Watershed Code MOR00).

2-year RI, 1-hour rainfall = $29 \mathrm{~mm}$. 10-year $\mathrm{RI}$, 1-hour rainfall $=42 \mathrm{~mm}$. 25-year $\mathrm{RI}$, 1-hour rainfall $=46 \mathrm{~mm}$.

\begin{tabular}{|c|c|c|c|c|c|c|c|c|c|}
\hline \\
\hline MOR00 & 23 & 28,000 & 42 & 35,000 & 49 & 37,000 & na & na & na \\
\hline MOR01 & 27 & 21,000 & 48 & 26,000 & 54 & 28,000 & 71 & 18 & 25 \\
\hline MOR02 & 22 & 3,800 & 42 & 4,700 & 49 & 5,000 & 102 & 70 & 89 \\
\hline MOR03 & 9 & 900 & 19 & 1,100 & 24 & 1,200 & 149 & 140 & 163 \\
\hline MOR30 & 36 & 3,200 & 58 & 4,000 & 65 & 4,200 & 21 & 77 & 29 \\
\hline
\end{tabular}

'Big Tooth Reservoir (South Ruxton Creek) Watershed (Primary Watershed Code BIG00).

2-year $\mathrm{RI}$, 1-hour rainfall = $29 \mathrm{~mm}$. 10-year RI, 1-hour rainfall $=42 \mathrm{~mm}$. 25-year RI, 1-hour rainfall = $46 \mathrm{~mm}$.

\begin{tabular}{rrrrrrrrr}
\hline BIG00 & 19 & 27,000 & 37 & 33,000 & 44 & 35,000 & na & na \\
BIG01 & 21 & 1,000 & 40 & 1,300 & 47 & 1,400 & 112 & 137 \\
BIG02 & 38 & 7,500 & 61 & 9,400 & 67 & 10,000 & 14 & 42 \\
BIG03 & 19 & 26,000 & 37 & 32,000 & 44 & 34,000 & 129 & 12 \\
BIG04 & 37 & 3,100 & 60 & 3,900 & 66 & 4,100 & 18 & 83 \\
BIG05 & 21 & 3,100 & 40 & 3,900 & 47 & 4,100 & 113 & 80 \\
BIG06 & 9 & 9,900 & 19 & 12,000 & 24 & 13,000 & 150 & 82 \\
\hline
\end{tabular}

${ }^{2}$ Manitou No. 1 Intake (Ruxton Creek) Watershed (Primary Watershed Code MAN00).

2-year RI, 1-hour rainfall = $29 \mathrm{~mm}$. 10-year RI, 1-hour rainfall $=42 \mathrm{~mm}$. 25-year Rl, 1-hour rainfall $=46 \mathrm{~mm}$.

\begin{tabular}{|c|c|c|c|c|c|c|c|c|c|}
\hline MAN00 & 20 & $>100,000$ & 39 & $>100,000$ & 46 & $>100,000$ & na & na & na \\
\hline MAN01 & 29 & 5,900 & 51 & 7,300 & 58 & 7,700 & 56 & 51 & 36 \\
\hline MAN02 & 14 & 1,500 & 29 & 1,900 & 35 & 2,000 & 139 & 121 & 142 \\
\hline MAN03 & 35 & 5,800 & 57 & 7,300 & 64 & 7,700 & 23 & 52 & 15 \\
\hline MAN04 & 44 & 8,600 & 67 & 11,000 & 72 & 11,000 & 2 & 36 & 6 \\
\hline MAN05 & 28 & 2,600 & 49 & 3,300 & 56 & 3,500 & 65 & 89 & 68 \\
\hline MAN06 & 32 & 1,400 & 54 & 1,800 & 61 & 1,900 & 40 & 125 & 82 \\
\hline MAN07 & 4 & 16,000 & 10 & 19,000 & 13 & 21,000 & 161 & 24 & 100 \\
\hline
\end{tabular}


Table 2. Debris-flow model input variables and estimated debris-flow probabilities and volumes for primary watersheds and subwatersheds.-Continued

[D M S, degrees, minutes, seconds; RI, recurrence interval: km², square kilometer; m/m, meter per meter; $\mathrm{m}^{3}$, cubic meter; mm, millimeter; na, not applicable; $<$, less than; >, greater than]

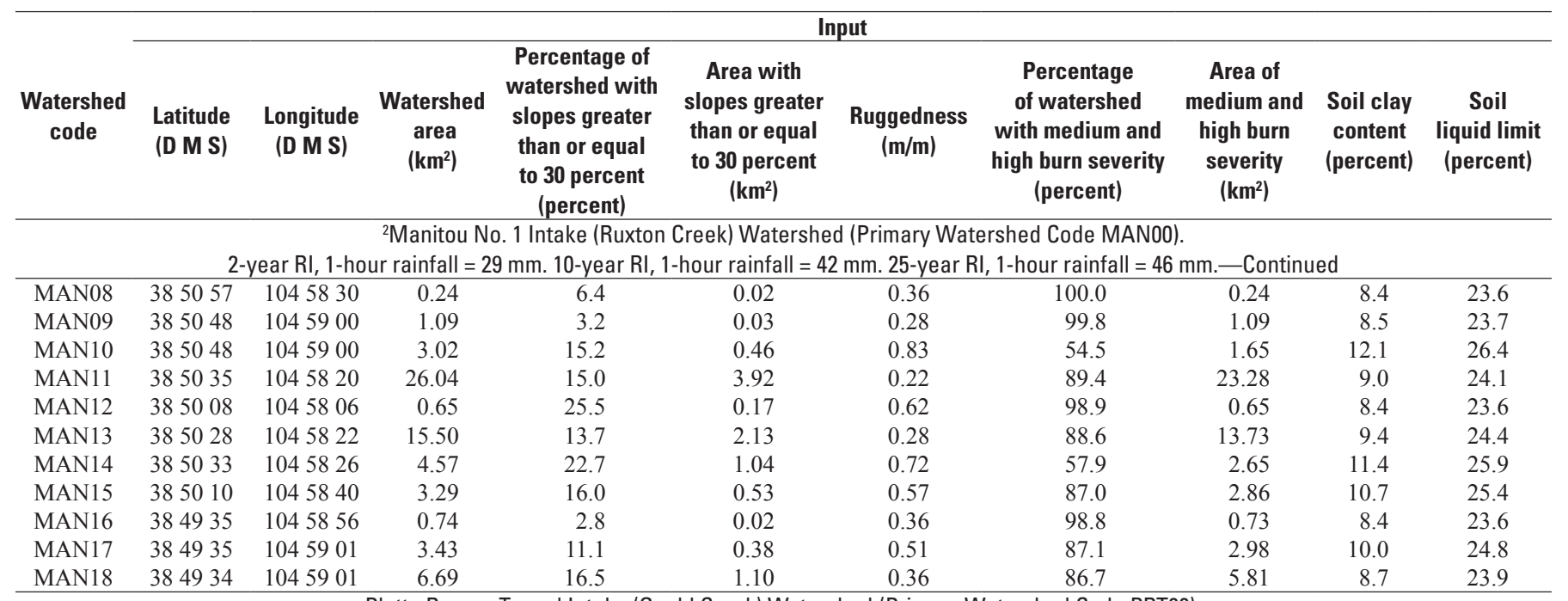

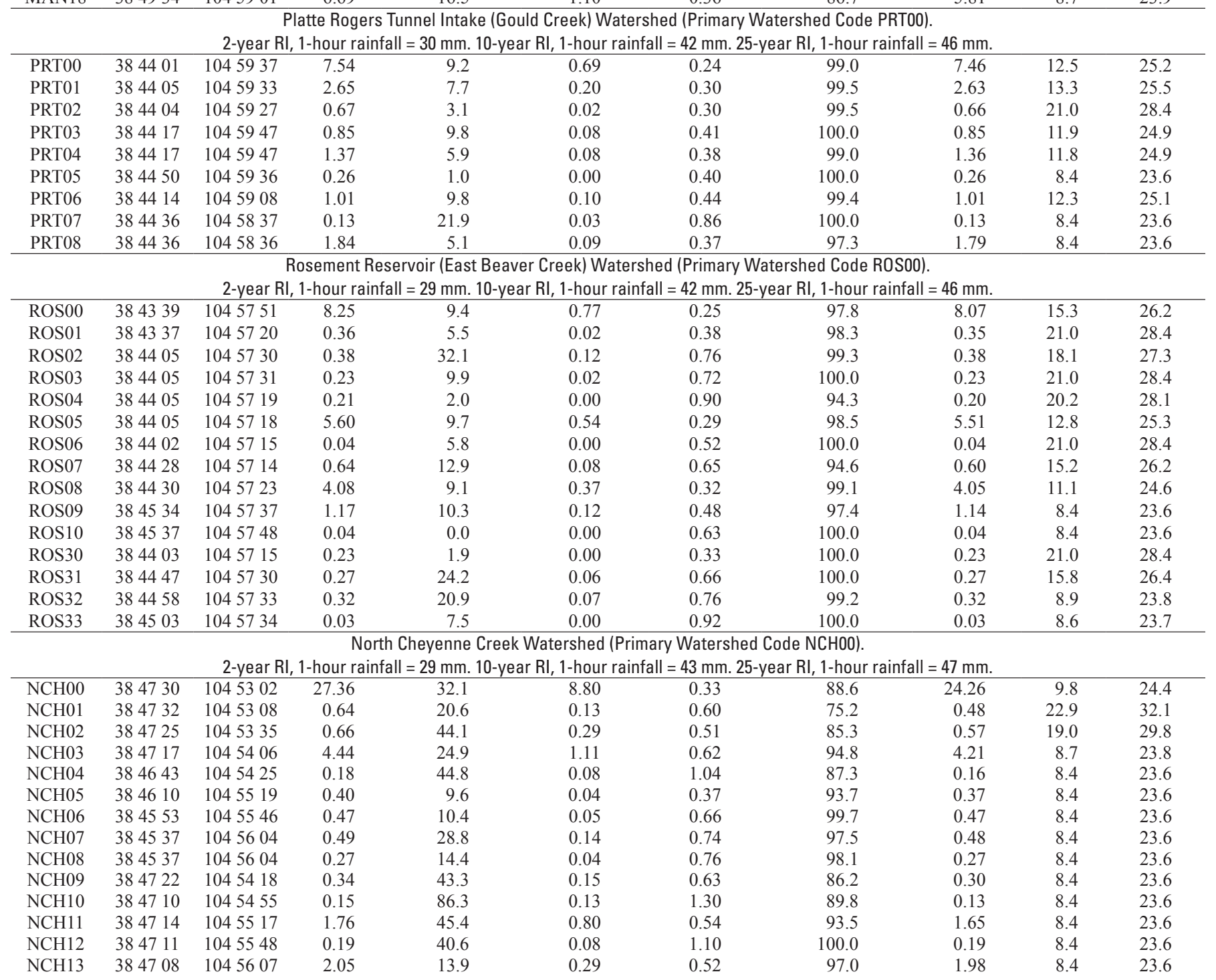


Table 2. Debris-flow model input variables and estimated debris-flow probabilities and volumes for primary watersheds and subwatersheds.-Continued

[DMS, degrees, minutes, seconds; RI, recurrence interval: $\mathrm{km}^{2}$, square kilometer; $\mathrm{m} / \mathrm{m}$, meter per meter; $\mathrm{m}^{3}$, cubic meter; mm, millimeter; na, not applicable; $<$, less than; >, greater than]

\begin{tabular}{|c|c|c|c|c|c|c|c|c|c|}
\hline \multirow[b]{2}{*}{$\begin{array}{l}\text { Watershed } \\
\text { code }\end{array}$} & \multicolumn{9}{|c|}{ Output } \\
\hline & $\begin{array}{c}\text { 2-year RI, } \\
\text { 1-hour rainfall } \\
\text { debris-flow } \\
\text { probability } \\
\text { (percent) }\end{array}$ & $\begin{array}{c}\text { 2-year RI, } \\
\text { 1-hour rainfall } \\
\text { debris-flow } \\
\text { volume } \\
\left(\mathrm{m}^{3}\right)\end{array}$ & $\begin{array}{c}\text { 10-year RI, } \\
\text { 1-hour rainfall } \\
\text { debris-flow } \\
\text { probability } \\
\text { (percent) }\end{array}$ & $\begin{array}{c}\text { 10-year RI, } \\
\text { 1-hour rainfall } \\
\text { debris-flow } \\
\text { volume } \\
\left(\mathrm{m}^{3}\right)\end{array}$ & $\begin{array}{c}\text { 25-year RI, } \\
\text { 1-hour rainfall } \\
\text { debris-flow } \\
\text { probability } \\
\text { (percent) }\end{array}$ & $\begin{array}{c}\text { 25-year RI, } \\
\text { 1-hour rainfall } \\
\text { debris-flow } \\
\text { volume } \\
\left(\mathrm{m}^{3}\right)\end{array}$ & $\begin{array}{c}\text { 25-year RI, } \\
\text { 1-hour rainfall } \\
\text { debris-flow } \\
\text { probability } \\
\text { rank }\end{array}$ & $\begin{array}{c}\text { 25-year RI, } \\
\text { 1-hour rainfall } \\
\text { debris-flow } \\
\text { volume rank }\end{array}$ & $\begin{array}{l}\text { 25-year RI, } \\
\text { 1-hour rainfall } \\
\text { combined } \\
\text { debris-flow } \\
\text { hazard rank }\end{array}$ \\
\hline
\end{tabular}

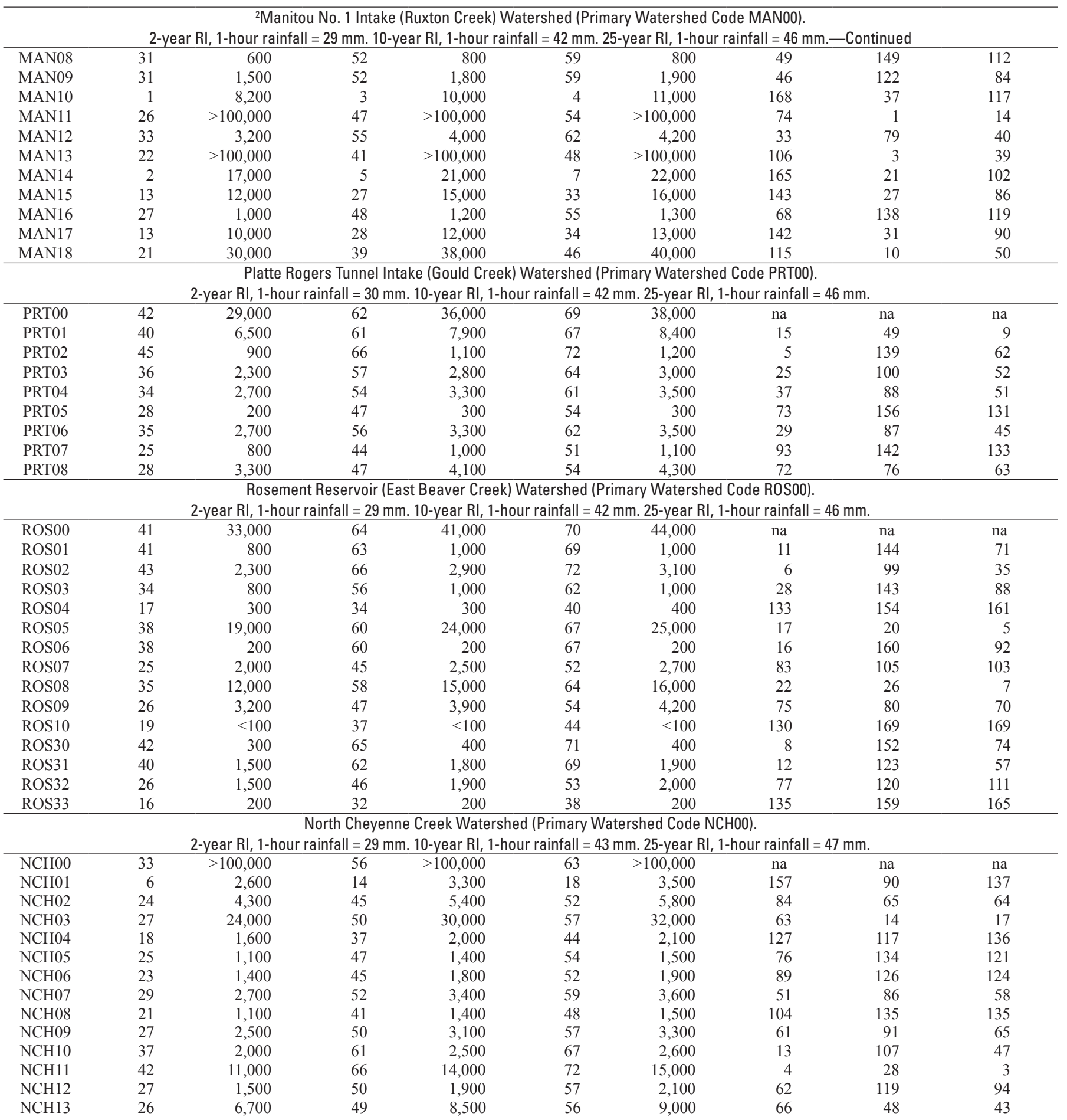




\section{A Prewildfire Evaluation for the Pikes Peak Area, El Paso and Teller Counties, Colorado}

Table 2. Debris-flow model input variables and estimated debris-flow probabilities and volumes for primary watersheds and subwatersheds.-Continued

[D M S, degrees, minutes, seconds; RI, recurrence interval: $\mathrm{km}^{2}$, square kilometer; $\mathrm{m} / \mathrm{m}$, meter per meter; $\mathrm{m}^{3}$, cubic meter; mm, millimeter; na, not applicable; $<$, less than; $>$, greater than]

\begin{tabular}{|c|c|c|c|c|c|c|c|c|c|c|}
\hline $\begin{array}{l}\text { Watershed } \\
\text { code }\end{array}$ & \multicolumn{10}{|c|}{ Input } \\
\hline \multicolumn{11}{|c|}{$\begin{array}{l}\text { North Cheyenne Creek Watershed (Primary Watershed Code NCH00). } \\
\text { fall }=29 \mathrm{~mm} .10 \text {-year RI, 1-hour rainfall = } 43 \mathrm{~mm} .25 \text {-year RI, 1-hour rainfall = } 47 \mathrm{~mm} \text {.-Continued }\end{array}$} \\
\hline NCH16 & 384621 & 1045710 & 0.25 & 48.2 & 0.12 & 0.85 & 98.7 & 0.25 & 8.4 & 23.6 \\
\hline NCH17 & 384623 & 1045717 & 1.30 & 22.8 & 0.30 & 0.57 & 96.6 & 1.25 & 8.4 & 23.6 \\
\hline NCH18 & 384551 & 1045719 & 0.71 & 9.7 & 0.07 & 0.53 & 93.7 & 0.66 & 8.4 & 23.6 \\
\hline NCH19 & 384626 & 1045726 & 3.26 & 25.8 & 0.84 & 0.49 & 93.1 & 3.04 & 8.4 & 23.6 \\
\hline $\mathrm{NCH} 20$ & 384618 & 1045748 & 1.67 & 24.1 & 0.40 & 0.59 & 90.1 & 1.51 & 8.4 & 23.6 \\
\hline $\mathrm{NCH} 21$ & 384612 & 1045749 & 0.47 & 12.3 & 0.06 & 0.43 & 98.4 & 0.46 & 8.4 & 23.6 \\
\hline $\mathrm{NCH} 30$ & 384708 & 1045424 & 4.21 & 23.6 & 0.99 & 0.56 & 94.8 & 4.00 & 8.4 & 23.6 \\
\hline \multicolumn{11}{|c|}{$\begin{array}{l}\text { South Cheyenne Creek Watershed (Primary Watershed Code SCH00). } \\
\text { r rainfall = } 30 \mathrm{~mm} .10 \text {-year RI, 1-hour rainfall = } 43 \mathrm{~mm} \text {. 25-year RI, 1-hour rainfall = } 48 \mathrm{~mm} \text {. }\end{array}$} \\
\hline $\mathrm{SCH} 00$ & 384708 & 1045217 & 25.78 & 30.8 & 7.93 & 0.30 & 94.5 & 24.37 & 17.9 & 29.0 \\
\hline $\mathrm{SCH} 01$ & 384655 & 1045249 & 0.33 & 68.6 & 0.23 & 0.77 & 68.6 & 0.23 & 22.9 & 32.1 \\
\hline $\mathrm{SCH} 02$ & 384637 & 1045251 & 0.87 & 32.9 & 0.28 & 0.60 & 99.7 & 0.86 & 22.9 & 32.1 \\
\hline $\mathrm{SCH} 03$ & 384556 & 1045252 & 0.63 & 42.0 & 0.26 & 0.78 & 99.0 & 0.62 & 22.9 & 32.1 \\
\hline $\mathrm{SCH} 04$ & 384556 & 1045252 & 1.60 & 46.7 & 0.75 & 0.52 & 99.9 & 1.60 & 22.9 & 32.1 \\
\hline $\mathrm{SCH} 05$ & 384552 & 1045305 & 0.87 & 9.6 & 0.08 & 0.38 & 100.0 & 0.87 & 22.9 & 32.1 \\
\hline SCH06 & 384525 & 1045312 & 0.20 & 7.5 & 0.02 & 0.52 & 100.0 & 0.20 & 22.9 & 32.1 \\
\hline $\mathrm{SCH} 07$ & 384521 & 1045312 & 0.51 & 26.7 & 0.14 & 0.80 & 100.0 & 0.51 & 22.9 & 32.1 \\
\hline $\mathrm{SCH} 08$ & 384512 & 1045315 & 0.77 & 33.7 & 0.26 & 0.69 & 99.8 & 0.77 & 22.9 & 32.1 \\
\hline $\mathrm{SCH} 18$ & 384631 & 1045325 & 1.07 & 13.0 & 0.14 & 0.53 & 91.4 & 0.98 & 11.9 & 25.7 \\
\hline SCH19 & 384558 & 1045355 & 1.18 & 9.8 & 0.12 & 0.56 & 99.1 & 1.17 & 13.6 & 26.6 \\
\hline $\mathrm{SCH} 20$ & 384554 & 1045413 & 0.70 & 46.0 & 0.32 & 0.81 & 92.0 & 0.64 & 8.4 & 23.6 \\
\hline $\mathrm{SCH} 21$ & 384537 & 1045430 & 1.01 & 53.8 & 0.54 & 0.61 & 83.1 & 0.84 & 8.4 & 23.6 \\
\hline $\mathrm{SCH} 22$ & 384518 & 1045443 & 1.64 & 34.3 & 0.56 & 0.79 & 92.9 & 1.52 & 11.2 & 24.7 \\
\hline $\mathrm{SCH} 23$ & 384438 & 1045605 & 0.41 & 27.2 & 0.11 & 0.90 & 83.3 & 0.34 & 19.2 & 27.7 \\
\hline $\mathrm{SCH} 24$ & 384438 & 1045605 & 0.55 & 21.1 & 0.12 & 0.63 & 95.9 & 0.53 & 21.0 & 28.4 \\
\hline $\mathrm{SCH} 30$ & 384517 & 1045443 & 3.44 & 38.3 & 1.32 & 0.53 & 90.2 & 3.10 & 14.2 & 25.8 \\
\hline
\end{tabular}


Table 2. Debris-flow model input variables and estimated debris-flow probabilities and volumes for primary watersheds and subwatersheds.-Continued

[DMS, degrees, minutes, seconds; RI, recurrence interval: $\mathrm{km}^{2}$, square kilometer; $\mathrm{m} / \mathrm{m}$, meter per meter; $\mathrm{m}^{3}$, cubic meter; mm, millimeter; na, not applicable; $<$, less than; >, greater than]

\begin{tabular}{|c|c|c|c|c|c|c|c|c|c|}
\hline \multirow[b]{2}{*}{$\begin{array}{l}\text { Watershed } \\
\text { code }\end{array}$} & \multicolumn{9}{|c|}{ Output } \\
\hline & $\begin{array}{l}\text { 2-year RI, } \\
\text { 1-hour rainfall } \\
\text { debris-flow } \\
\text { probability } \\
\text { (percent) }\end{array}$ & $\begin{array}{c}\text { 2-year RI, } \\
\text { 1-hour rainfall } \\
\text { debris-flow } \\
\text { volume } \\
\left(\mathrm{m}^{3}\right)\end{array}$ & $\begin{array}{l}\text { 10-year RI, } \\
\text { 1-hour rainfall } \\
\text { debris-flow } \\
\text { probability } \\
\text { (percent) }\end{array}$ & $\begin{array}{c}10 \text {-year RI, } \\
\text { 1-hour rainfall } \\
\text { debris-flow } \\
\text { volume } \\
\left(\mathrm{m}^{3}\right)\end{array}$ & $\begin{array}{l}\text { 25-year RI, } \\
\text { 1-hour rainfall } \\
\text { debris-flow } \\
\text { probability } \\
\text { (percent) }\end{array}$ & $\begin{array}{l}\text { 25-year RI, } \\
\text { 1-hour rainfall } \\
\text { debris-flow } \\
\text { volume } \\
\left(\mathrm{m}^{3}\right)\end{array}$ & $\begin{array}{c}\text { 25-year RI, } \\
\text { 1-hour rainfall } \\
\text { debris-flow } \\
\text { probability } \\
\text { rank }\end{array}$ & $\begin{array}{c}\text { 25-year RI, } \\
\text { 1-hour rainfall } \\
\text { debris-flow } \\
\text { volume rank }\end{array}$ & $\begin{array}{c}\text { 25-year RI, } \\
\text { 1-hour rainfall } \\
\text { combined } \\
\text { debris-flow } \\
\text { hazard rank }\end{array}$ \\
\hline \multicolumn{10}{|c|}{$\begin{array}{l}\text { North Cheyenne Creek Watershed (Primary Watershed Code NCH00). } \\
\text { all = } 29 \mathrm{~mm} .10 \text {-year Rl, 1-hour rainfall = } 43 \mathrm{~mm} .25 \text {-year Rl, 1-hour rainfall = } 47 \mathrm{~mm} \text {. - Continued }\end{array}$} \\
\hline NCH16 & 40 & 2,100 & 64 & 2,700 & 70 & 2,800 & 10 & 103 & 42 \\
\hline $\mathrm{NCH} 17$ & 30 & 5,600 & 53 & 7,100 & 60 & 7,500 & 45 & 53 & 28 \\
\hline $\mathrm{NCH} 18$ & 20 & 1,900 & 41 & 2,400 & 48 & 2,500 & 109 & 109 & 126 \\
\hline $\mathrm{NCH} 19$ & 30 & 16,000 & 53 & 20,000 & 60 & 22,000 & 43 & 22 & 10 \\
\hline $\mathrm{NCH} 20$ & 22 & 7,300 & 43 & 9,200 & 50 & 9,700 & 97 & 44 & 61 \\
\hline $\mathrm{NCH} 21$ & 30 & 1,500 & 54 & 2,000 & 60 & 2,100 & 41 & 118 & 73 \\
\hline $\mathrm{NCH} 26$ & 28 & 1,900 & 51 & 2,500 & 58 & 2,600 & 57 & 108 & 81 \\
\hline $\mathrm{NCH} 30$ & 28 & 21,000 & 51 & 27,000 & 58 & 29,000 & 54 & 16 & 11 \\
\hline \multicolumn{10}{|c|}{ South Cheyenne Creek Watershed (Primary Watershed Code SCHOO). } \\
\hline SCH00 & 38 & $>100,000$ & 60 & $>100,000$ & 68 & $>100,000$ & na & na & na \\
\hline $\mathrm{SCH} 01$ & 13 & 3,100 & 27 & 3,900 & 34 & 4,200 & 141 & 81 & 127 \\
\hline $\mathrm{SCH} 02$ & 30 & 4,900 & 51 & 6,100 & 60 & 6,500 & 44 & 59 & 34 \\
\hline $\mathrm{SCH} 03$ & 29 & 4,200 & 50 & 5,200 & 59 & 5,600 & 52 & 67 & 46 \\
\hline $\mathrm{SCH} 04$ & 43 & 11,000 & 65 & 14,000 & 72 & 15,000 & 1 & 30 & 2 \\
\hline $\mathrm{SCH} 05$ & 23 & 2,400 & 43 & 2,900 & 52 & 3,100 & 88 & 96 & 99 \\
\hline SCH06 & 19 & 600 & 36 & 700 & 45 & 800 & 123 & 150 & 155 \\
\hline SCH07 & 21 & 2,700 & 39 & 3,300 & 48 & 3,600 & 107 & 85 & 106 \\
\hline $\mathrm{SCH} 08$ & 28 & 4,400 & 49 & 5,500 & 57 & 5,900 & 59 & 62 & 48 \\
\hline $\mathrm{SCH} 18$ & 19 & 3,300 & 37 & 4,100 & 45 & 4,400 & 120 & 75 & 108 \\
\hline SCH19 & 23 & 3,100 & 43 & 3,900 & 52 & 4,200 & 90 & 78 & 83 \\
\hline $\mathrm{SCH} 20$ & 32 & 4,800 & 54 & 6,000 & 62 & 6,400 & 27 & 60 & 23 \\
\hline $\mathrm{SCH} 21$ & 32 & 7,100 & 54 & 8,800 & 63 & 9,500 & 26 & 45 & 12 \\
\hline $\mathrm{SCH} 22$ & 29 & 9,100 & 51 & 11,000 & 59 & 12,000 & 48 & 34 & 20 \\
\hline $\mathrm{SCH} 23$ & 19 & 2,200 & 36 & 2,700 & 45 & 2,900 & 121 & 102 & 128 \\
\hline $\mathrm{SCH} 24$ & 41 & 2,500 & 63 & 3,100 & 71 & 3,300 & 9 & 93 & 33 \\
\hline $\mathrm{SCH} 30$ & 41 & 22,000 & 63 & 27,000 & 71 & 29,000 & 7 & 15 & 1 \\
\hline
\end{tabular}

${ }^{1}$ Lake Moraine and Big Tooth Reservoir are located within the greater Ruxton Creek watershed and upstream from the Manitou \#1 Intake.

${ }^{2}$ Ruxton Creek main-stem (MAN00) probability and volume estimates include the areas upstream from Lake Moraine (MOR00) and Big Tooth Reservoir (BIG00); however, model assumes no reservoir effect. Ruxton Creek drainage area at MAN00 is greater than the maximum drainage area that produced a debris flow in Cannon and others, $2010\left(30 \mathrm{~km}^{2}\right)$. See text p. 4 for explanation. 
Three postwildfire precipitation scenarios and recurrence intervals were used for the postwildfire debris-flow analysis in the Pikes Peak area watersheds. These scenarios were (1) a 2-year recurrence (50-percent annual exceedance probability), 1-hour duration rainfall; (2) a 10-year recurrence (10-percent annual exceedance probability), 1-hour duration rainfall; and (3) a 25-year recurrence (4-percent annual exceedance probability), 1-hour duration rainfall. In this report, the precipitation scenarios will be referred to as the " $2-, 10$-, and 25-year storms." Rainfall totals for the Pikes Peak area watersheds were extrapolated from the National Oceanic and Atmospheric Administration (NOAA) Atlas II for Colorado (Miller and others, 1973). A 1-hour rainfall duration was chosen for the scenarios because it is a relatively short-lived event, and because it was the longest rainfall period for which rainfall intensity was calculated when the debris-flow models were derived (Cannon and others, 2010, their table 2). The total storm rainfall for each scenario was assumed to occur uniformly over each primary watershed.

Other input variables for the debris-flow model were determined from a variety of sources. The watershed area and percentage of watershed area with hillslopes of 30 percent or greater were determined using ArcMap (Environmental Systems Research Institute, Inc., 2009) with topography from 10-m digital-elevation models (DEMs) (Gesch and others, 2002). Raw data for soil properties were compiled from the State Soil Geographic (STATSGO) database (U.S. Department of Agriculture, 1991), which was processed by Schwartz and Alexander (1995) to obtain soil clay content and liquid limit. Soil properties were spatially averaged when more than one value occurred in a watershed. Because tools were not available in the standard ArcGIS toolbox to evaluate ruggedness in a spatially explicit manner, a python script was written to evaluate the ruggedness variable for each grid cell in the study area.

\section{Watershed Characterization}

Debris-flow probabilities and volumes for this study were estimated using two watershed-characterization methods. First, a conventional watershed-characterization approach was used. Secondly, the debris-flow probabilities and volumes were estimated using a continuous-parameterization technique (Verdin and Greenlee, 2003; Verdin and Worstell, 2008).

For the conventional watershed characterization approach, 14 primary watersheds (identified by Colorado Springs Utilities) and 170 selected subwatersheds within the primary watersheds (tables 1 and 2) were delineated using Streamstats (U.S. Geological Survey, 2010). The 170 subwatersheds were selected based on (1) topographic characteristics and vegetation distribution as displayed in the Streamstats interface, and (2) geomorphic field evidence of previous debris flows. Watershed sizes in the analysis ranged from 0.01 to $42.65 \mathrm{~km}^{2}\left(0.004\right.$ to $16.47 \mathrm{mi}^{2}$ ) (table 2), which is consistent with the range of watershed areas used in the debris-flow models developed by Cannon and others (2010), 0.01 to $103 \mathrm{~km}^{2}$ (0.004 to $39.77 \mathrm{mi}^{2}$ ). The 14 primary watersheds and 170 subwatersheds were evaluated by averaging the input variables over the watershed area and using those values in the debris-flow equations.

Whereas the conventional watershed-characterization method allows evaluation of the debris-flow probability and volume equations at predefined locations only (generally at the watershed outlet), the continuous-parameterization technique, using the 1/3-arc-second National Elevation Dataset (Gesch and others, 2002) (10-m nominal resolution) and its derived flow-direction grid as a base, evaluates the debris-flow equations for every pixel within the 10-m DEM. This technique provides a synoptic view of the entire study area, providing estimates of debris-flow volume and probability in a continuous manner for the entire channel length within a watershed. Examination of the derived probabilities and volumes along all stream channels facilitates identification of areas of high or low potential for debris flows.

Evaluation of the debris-flow equations using the continuous-parameterization technique requires that surfaces of all of the independent variables used as input to the predictive equations be developed. Through use of the flow-direction grid and techniques detailed in Verdin and Worstell (2008), surfaces were developed for all of the independent variables. Once the surfaces of the independent variables were developed, the probability and volume equations were solved using map algebra for each grid cell and the 2-year, 10-year, and 25 -year storms. Identification of the probability or volume of a debris flow at any location within the study area is possible by querying the derived surfaces. In this assessment, a raster sampling technique was used to identify the values of debrisflow probability and volume at selected locations along the drainage network derived from a digital-elevation model. The results from the continuous-parameterization approach were identical to the results of the conventional watershedcharacterization approach at the watershed outlet, or pour point, of the 14 primary watersheds and 170 subwatersheds defined within the study area. The advantage of the continuous-parameterization technique is that it provides the capability to rapidly evaluate and assess subwatershed probability and volume estimates at specific drainage-network locations within a watershed, as well as at the watershed outlet.

\section{Verification of Debris-Flow Model Results}

Preliminary estimates of debris-flow probability made with the Cannon and others (2010) model were checked against geomorphic evidence onsite for selected watersheds in the study area during a reconnaissance visit. The presence of older debris-flow deposits or debris-flow-scoured channels in these watersheds was considered to be geomorphic evidence that debris flows had occurred at some time in the past, and that the debris-flow models of Cannon and others (2010) were 
appropriate for use in the Pikes Peak area watersheds. The purpose of the reconnaissance was to verify historical debrisflow activity, and no attempt was made to determine what watershed conditions (postwildfire or unburned) existed at the time the debris flows occurred.

The debris-flow probability model was run for each watershed with the assumptions that (1) all trees and shrubs in the watershed had been burned with a moderate to high severity, and that the fire soon was followed by (2) a 2-year recurrence, 1-hour storm. This scenario represented a relatively rare wildfire (a moderate- to high-burn severity of the entire watershed) and a relatively common rainfall (50-percent annual-exceedance probability) likely to occur soon after a wildfire.

Debris-flow probabilities for all watersheds in the study area ranged from less than 1 to 46 percent for the assumed burn severity and extent and the assumed 2-year storm. USGS personnel performed reconnaissance and onsite verification in 47 study-area watersheds in August 2010. Forty-two of these watersheds had an estimated debris-flow probability of 20 percent or greater as a result of the assumed burn severity and extents followed by a 2-year storm. Eighteen of these 42 high-probability watersheds (43 percent) and one watershed with a 19-percent probability (SCH-13) showed geomorphic evidence of previous debris-flow activity including lateral, or marginal, levees (figs. 2 and 3), terminal lobes, debris-flow fans, or debris-flow-scoured channels (Costa, 1988; Pierson, 2005). Fourteen of these watersheds (33 percent) had inconclusive geomorphic evidence; such as lobe-shaped deposits of colluvium or reworked glacial till that could have been formed by hillslope creep, landslide, earthflow, solifluction, rockfall, debris flow, or combinations thereof (Keefer and Johnson, 1983). Ten of these watersheds (24 percent) showed no geomorphic evidence of previous debris-flow activity.

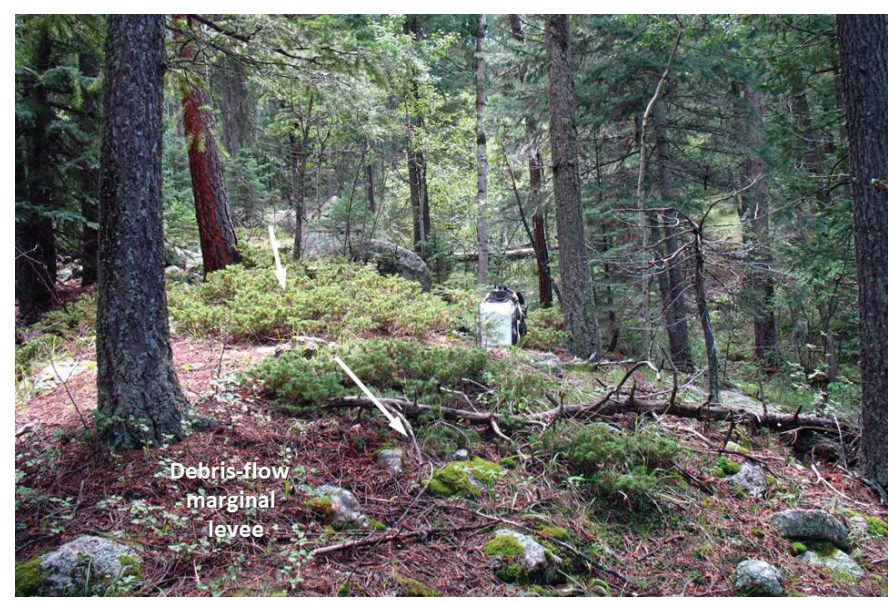

Figure 2. Debris-flow marginal levee near the outlet of subwatershed SCH13, a tributary in the South Cheyenne Creek watershed. View is looking upstream; arrows indicate direction of debris flow. Photograph by Keelin R. Schaffrath, U.S. Geological Survey, August 24, 2010.

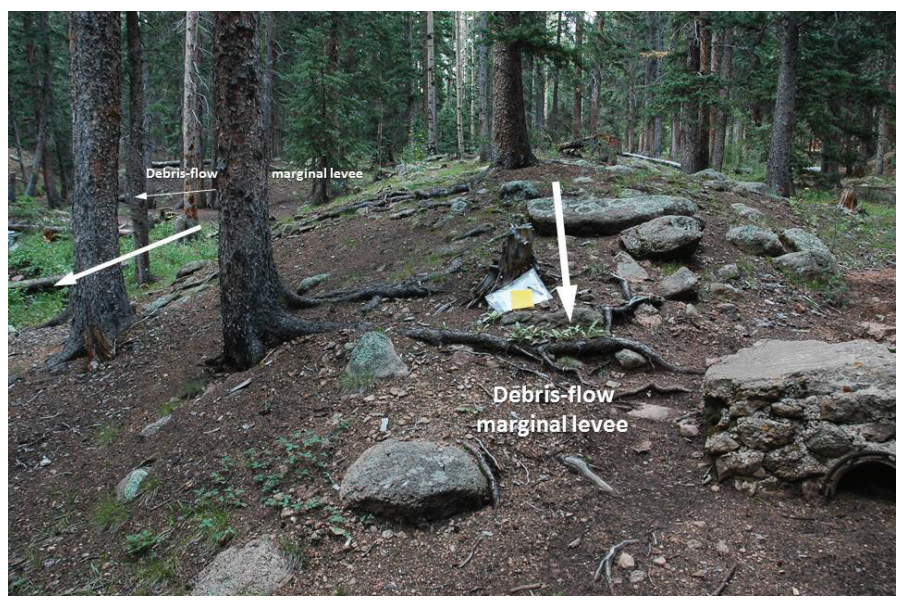

Figure 3. Debris-flow marginal levees bordering East Beaver Creek in subwatershed ROS08, a tributary that flows into the Penrose-Rosemont Reservoir. View is looking upstream; arrows indicate direction of debris flow. Photograph by John G. Elliott, U.S. Geological Survey, August 25, 2010.

No attempt was made to correlate any observed debrisflow deposit with a previous wildfire, a specific storm characteristic, or a date. However, in watersheds for which the model predicted a greater than 20-percent probability of debris-flow activity for the 2-year storm, corroborative geomorphic evidence typically was found. The geomorphic evidence of debris-flow activity was subtle in most observed watersheds and debris-flow deposits commonly were found in heavily forested locations, indicating that the most recent debrisflow activity was at least several decades old (figs. 2 and 3). Although it was not determined whether any of the observed debris flows in the reconnaissance subwatersheds were the result of previous wildfires, the field evidence indicated that debris-flow processes had been active in these locations. Therefore, it was concluded that the Cannon and others (2010) models were appropriate to estimate the probability and volume of postwildfire debris flows in these watersheds for a range of postwildfire rainfall scenarios.

\section{Estimated Probabilities and Volumes of Postwildfire Debris Flows}

Potential postwildfire debris-flow probabilities and volumes for 14 primary watersheds and 170 subwatersheds located within the primary watersheds were estimated by using the empirical debris-flow models of Cannon and others (2010), equations 1,2 , and 3 . The debris-flow models assumed that a moderate to severe wildfire burned 100 percent of the forest and shrub stands within the watershed, and that rainstorms occurred within 4 to 6 years following the hypothetical wildfire (Elliott and others, 2005). Three postwildfire precipitation 
scenarios were used to represent a range of precipitation scenarios that could occur shortly after a wildfire in the Pikes Peak region: (1) a 2-year recurrence, 1-hour duration rainfall (2-year storm); (2) a 10-year recurrence, 1-hour duration rainfall (10-year storm); and (3) a 25-year recurrence, 1-hour duration rainfall (25-year storm).

The estimated probabilities and volumes are hypothetical and have been made due to the need for timely best science information. The estimates are provided on the condition that neither the U.S. Geological Survey nor the United States Government may be held liable for any damages resulting from the authorized or unauthorized use of the estimates.

\section{Pikes Peak Area Watershed Debris-Flow Probabilities}

Results of the debris-flow probability modeling are shown graphically as color-coded map symbols at primary watershed and subwatershed outlets in figures 4, 5, and 6; the corresponding numerical values are presented in table 2 . The color-coded map symbols represent the probability of a debris flow occurring in the channel at the primary watershed or subwatershed outlet estimated using the conventional watershed-characterization approach discussed in the "Watershed Characterization" section.

In addition to the color coded map symbols, the maps in figures 4, 5, and 6 include color-coded shaded areas representing the debris-flow probabilities in third-order streams in the study area. Stream order is a method of classifying the components of the drainage network and is a measure of the position of a stream in the hierarchy of tributaries within a watershed (Horton, 1945). The Strahler order (Strahler, 1957) is one such method and was used in this analysis. Strahler third-order watersheds were assessed for debris-flow probability using the continuous-parameterization technique described in the "Watershed Characterization" section. Although the contributing area upstream from the outlet of each Strahler third-order watershed is entirely shaded by a color representing the debris-flow estimated probability, the estimated probability is applicable only for a debris flow occurring at the point at which smaller channels converge to form a Strahler third-order channel, and not for every channel segment upstream from that point. The shaded areas give a detailed breakdown of the debris-flow estimated probability in smaller areas of the subwatersheds, providing useful information for resource managers and emergency responders.

The estimated probabilities for postwildfire debris flows in the 170 subwatersheds in the Pikes Peak study area ranged from less than 1 to 46 percent in response to the 2-year storm, 1 to 67 percent in response to the 10 -year storm, and 1 to 72 percent in response to the 25 -year storm (table 2). Subwatersheds with the lowest postwildfire debris-flow probabilities tended to have large areas of alpine and subalpine vegetation or other large areas with sparse forest cover (figs. 4-6).
Subwatersheds with the highest probabilities tended to be heavily forested and tended to have a large percent area of steep slopes (table 2). Forty of the 170 subwatersheds had a greater than 60 percent probability of producing a debris flow in response to the 25-year storm (fig. 6 and table 2). The drainage areas of these 40 high-probability subwatersheds ranged from 0.04 to $10.83 \mathrm{~km}^{2}$ and averaged $1.38 \mathrm{~km}^{2}$. Cannon and others (2010) found that "low-order tributaries" with a mean area of $1.7 \mathrm{~km}^{2}$ produced most of the debris flows in their study areas. Many of the subwatersheds with the highest debris-flow probabilities in this study were in the eastern and southeastern part of the study area, notably tributaries in the Gould Creek (Platte Rogers Tunnel Intake, PRT), East Beaver Creek (Rosemont Reservoir, ROS), North Cheyenne Creek (NCH), and South Cheyenne Creek (SCH) primary watersheds (fig. 6 and table 2).

The 14 primary watersheds were evaluated separately because they consisted of nested subwatersheds, and any potential debris flow reaching the primary watershed outlet could be the result of debris-flow contributions from the nested subwatersheds and also could include runoff from other contributing land surfaces (for example, laterally planar hillslopes) within the primary watershed (figs. 4, 5, 6). Postwildfire debris-flow probabilities for the primary watersheds ranged from 4 to 42 percent in response to the 2 -year storm, 8 to 64 percent in response to the 10 -year storm, and 10 to 70 percent in response to the 25-year storm (table 2).

The PRT, ROS, NCH, and SCH primary watersheds each had a greater than 60-percent probability of producing a debris flow at the watershed outlet in response to a 25 -year storm (table 2) if the entire forested part of the watershed was moderately to severely burned. The PRT and ROS primary watersheds had a greater than 40 -percent probability of producing a debris flow in response to as little as a 2-year storm.

It is possible for a large primary watershed to have a very small percent probability of a debris flow reaching the watershed outlet even though debris flows were possible in some subwatersheds within the primary watershed This possibility occurs in some primary watersheds because of limited transport potential downstream from a subwatershed outlet or because of the relatively small size of the debris-flow contributing area within the primary watershed. Cannon and others (2010) found that "debris flows were not observed at the outlets of watersheds greater than about $30 \mathrm{~km}^{2}\left(12 \mathrm{mi}^{2}\right)$ in area."

\section{Pikes Peak Area Watershed Debris-Flow Volumes}

Results of the debris-flow volume models are shown graphically as color-coded map symbols at primary watershed and subwatershed outlets in figures 7,8, and 9: the corresponding numerical values are presented in tables 2 . The color-coded map symbols represent the estimated volume of a debris flow occurring in the channel at the primary watershed or subwatershed outlet estimated using the conventional watershed-characterization approach discussed in the "Watershed Characterization" section. 

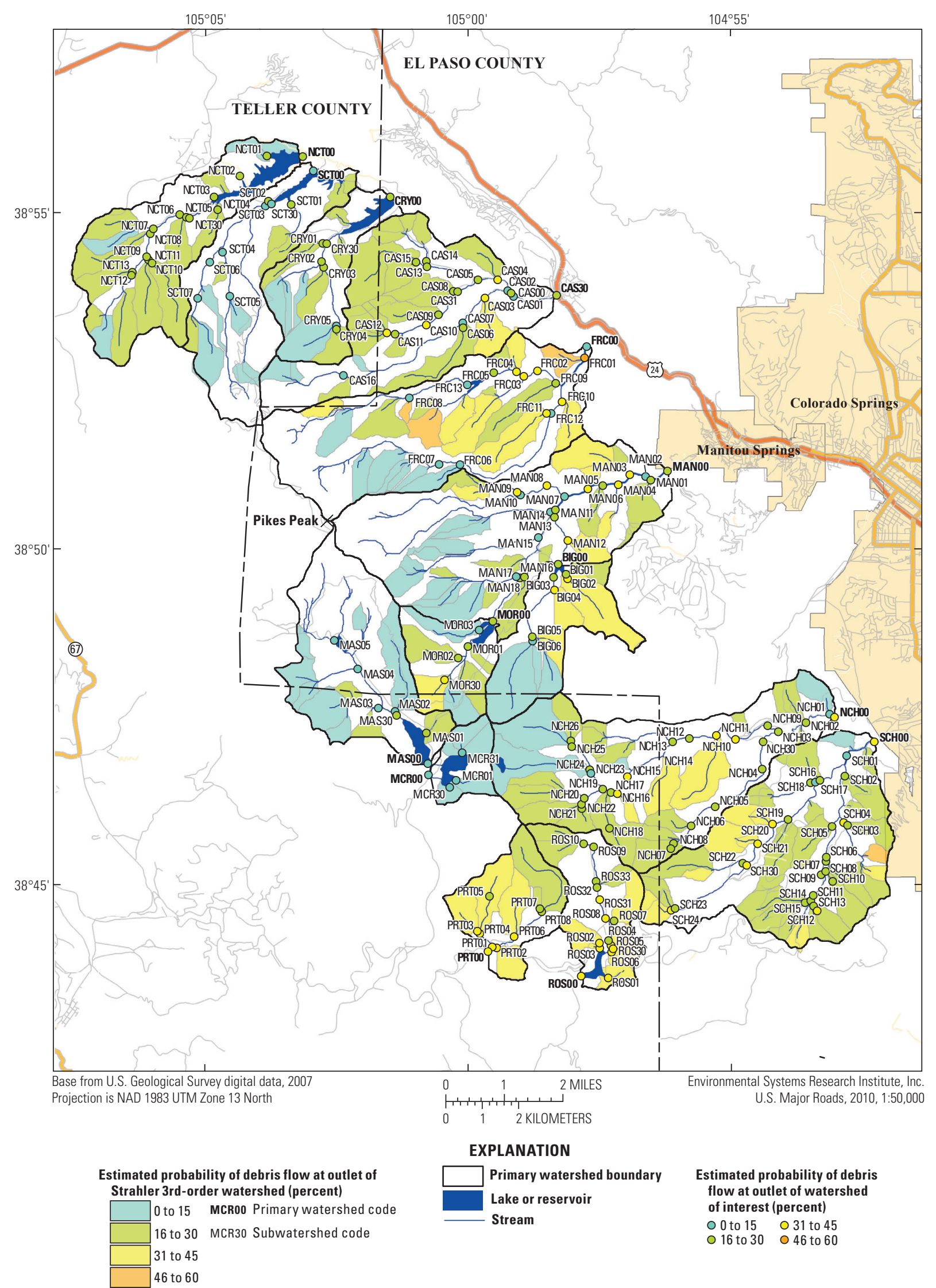

Figure 4. Map of primary watersheds and subwatersheds showing estimated debris-flow probabilities in response to the 2-year-recurrence, 1-hour-duration rainfall. See table 2 for watershed and subwatershed codes. 


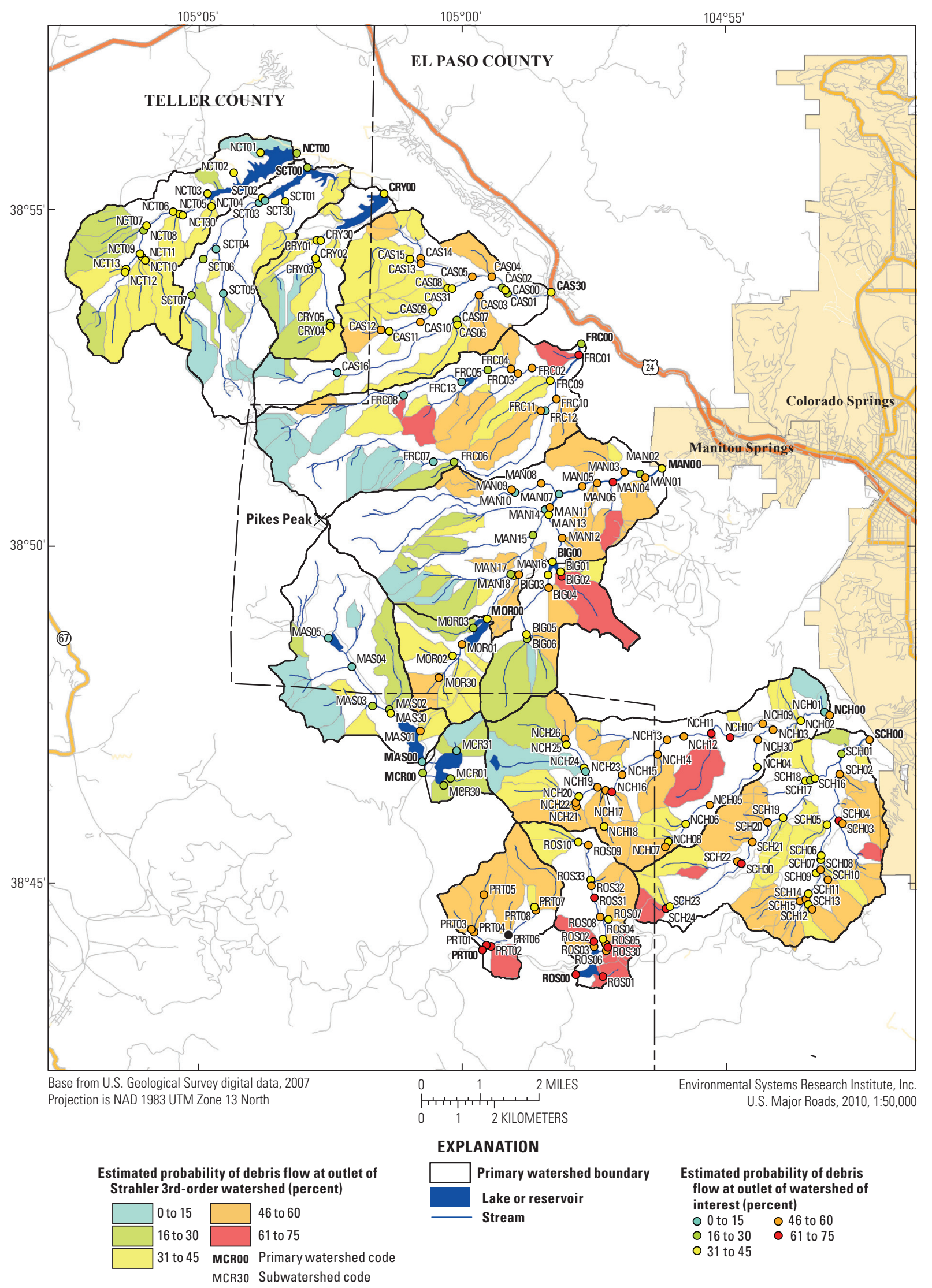

Figure 5. Map of primary watersheds and subwatersheds showing estimated debris-flow probabilities in response to the 10-year-recurrence, 1 -hour-duration rainfall. See table 2 for watershed and subwatershed codes. 


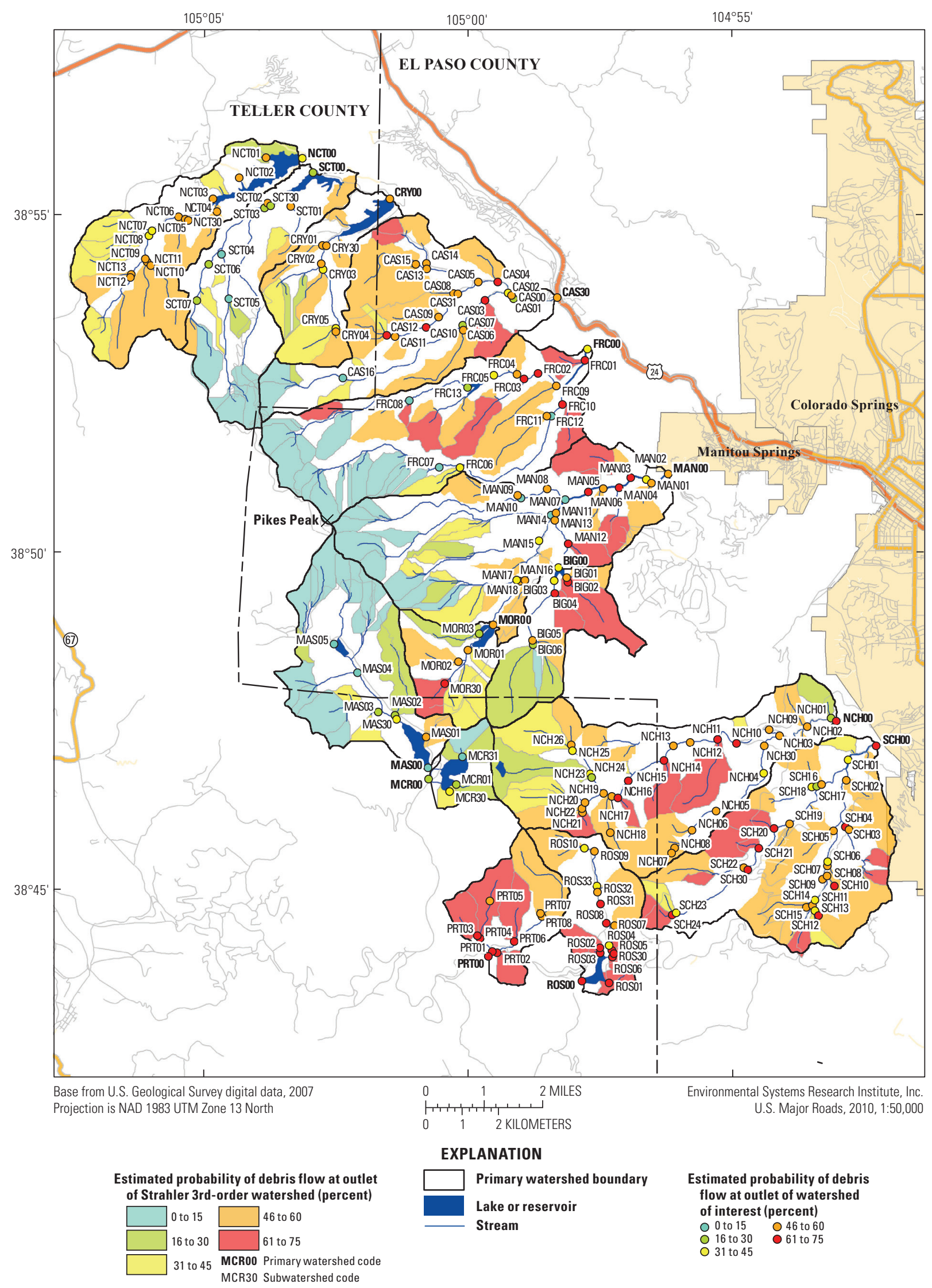

Figure 6. Map of primary watersheds and subwatersheds showing estimated debris-flow probabilities in response to the 25-year-recurrence, 1-hour-duration rainfall. See table 2 for watershed and subwatershed codes. 


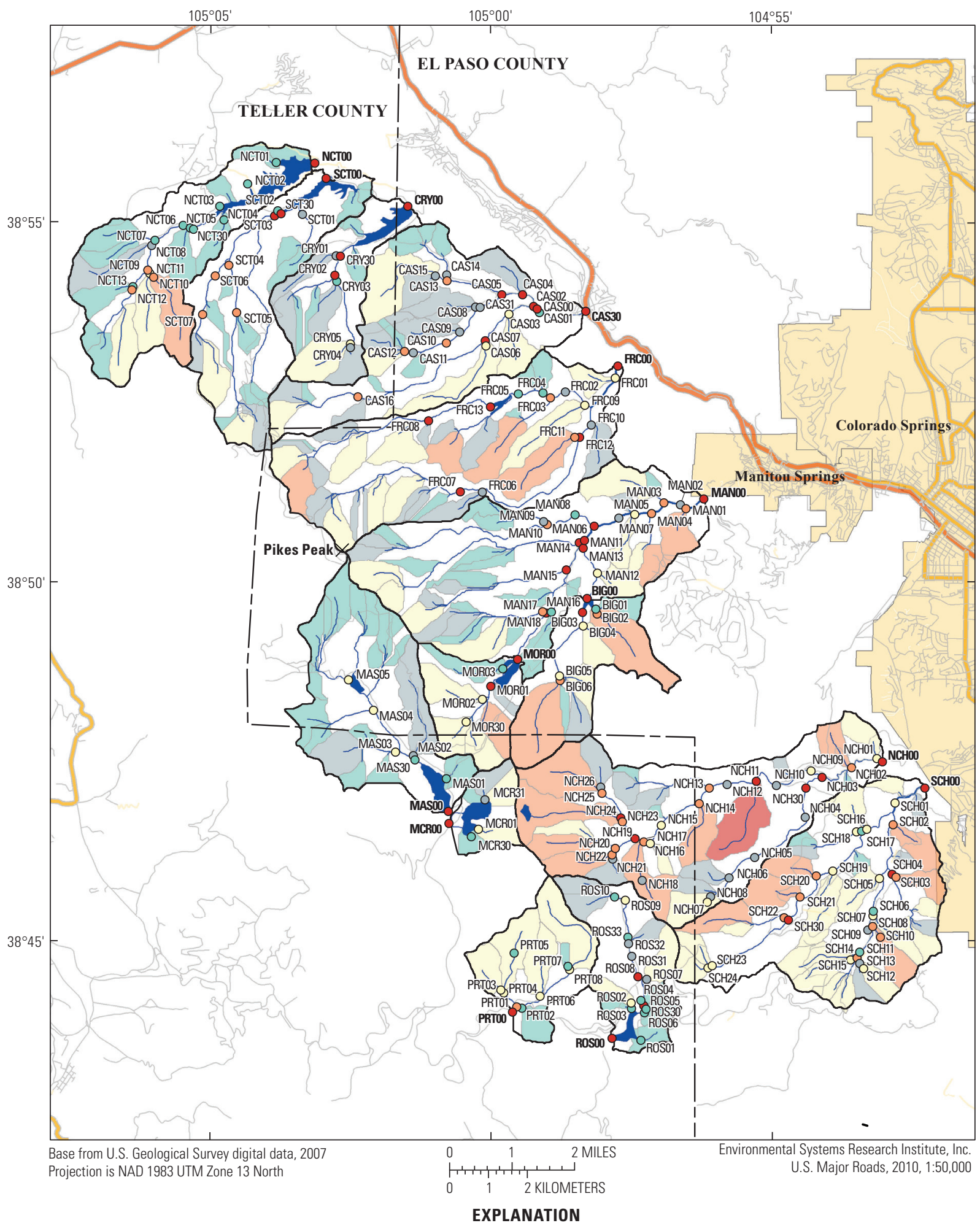

\begin{tabular}{|c|c|c|c|}
\hline \multirow{2}{*}{\multicolumn{2}{|c|}{$\begin{array}{l}\text { Estimated volume of debris flow at outlet of Strahler 3rd-order } \\
\text { watershed (cubic meters) }\end{array}$}} & & \multirow{3}{*}{$\begin{array}{l}\text { Estimated volume of debris flow } \\
\text { at outlet of watershed of interest } \\
\text { (cubic meters) }\end{array}$} \\
\hline & & \multirow{6}{*}{$\begin{array}{l}\text { Primary watershed boundary } \\
\text { Lake or reservoir } \\
\text { Stream }\end{array}$} & \\
\hline Less than 1,000 & MCR00 Primary watershed code & & \\
\hline Greater than 1,000 to 2,000 & MCR30 Subwatershed code & & O Less than 1,000 \\
\hline Greater than 2,000 to 4,000 & & & O Greater than 1,000 to 2,000 \\
\hline Greater than 4,000 to 10,000 & & & - Greater than 4,000 to 10,000 \\
\hline Greater than 10,000 & & & - Greater than 10,000 \\
\hline
\end{tabular}

Figure 7. Map of primary watersheds and subwatersheds showing estimated debris-flow volumes in response to the 2-yearrecurrence, 1-hour-duration rainfall. See table 2 for watershed and subwatershed codes. 


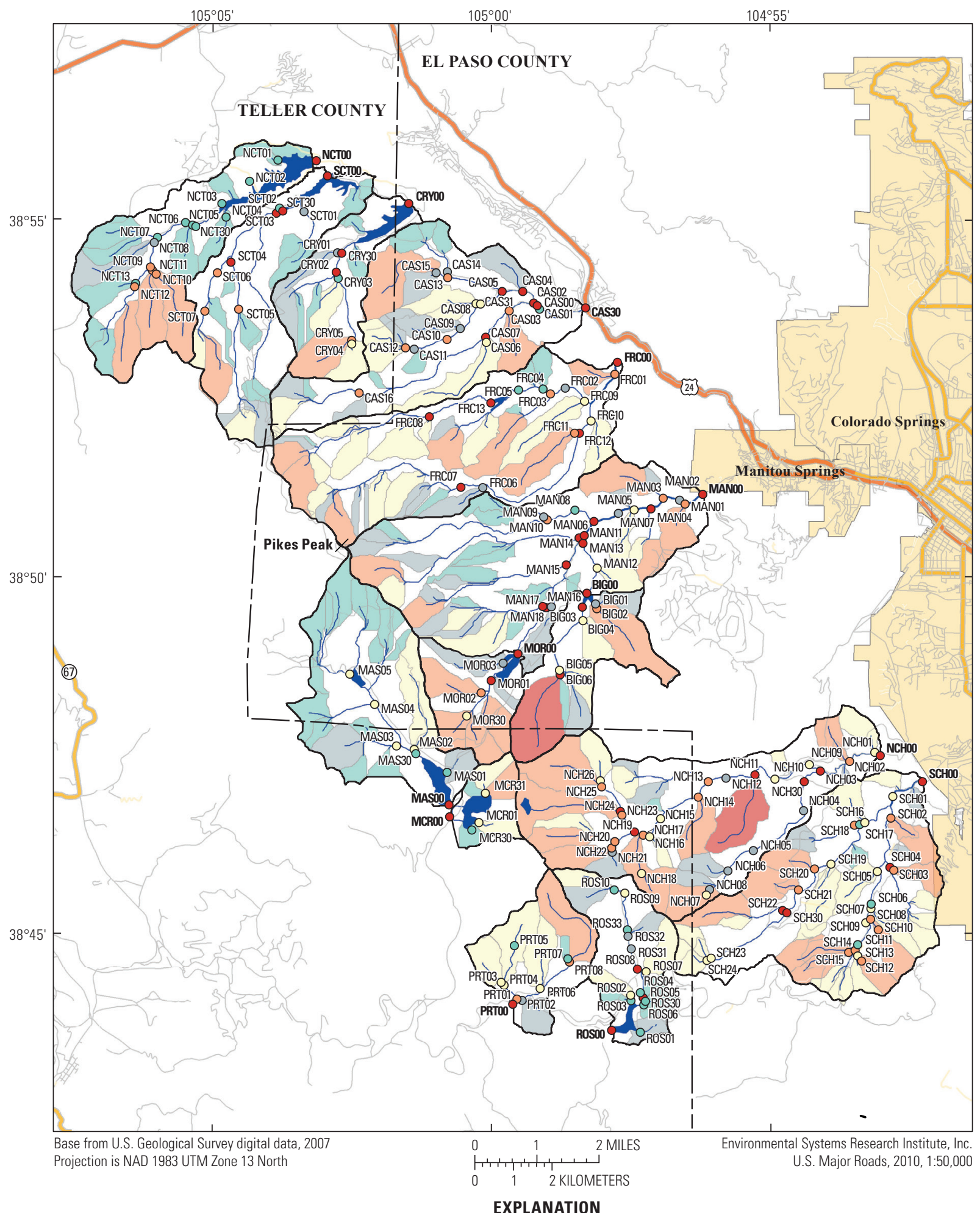

Estimated volume of debris flow at outlet of Strahler 3rd-order watershed (cubic meters)

\begin{tabular}{l}
\hline \\
\begin{tabular}{|l}
\hline \\
\hline
\end{tabular} Gess than $1,000 \quad$ MCR00 Primary watershed code \\
Greater than 2,000 to 4,000 \\
Greater than 4,000 to 10,000 \\
Greater than 10,000
\end{tabular}

\begin{tabular}{|ll}
\hline Primary watershed boundary & $\begin{array}{c}\text { Estimated volume of debris flow } \\
\text { at outlet of watershed of interest } \\
\text { Lake or reservoir }\end{array}$ \\
(cubic meters) \\
Stream & O Less than 1,000 \\
& $\circ$ Greater than 1,000 to 2,000 \\
& $\circ$ Greater than 2,000 to 4,000 \\
& $\circ$ Greater than 4,000 to 10,000 \\
& $\circ$ Greater than 10,000
\end{tabular}

Figure 8. Map of primary watersheds and subwatersheds showing estimated debris-flow volumes in response to the 10-year-recurrence, 1-hour-duration rainfall. See table 2 for watershed and subwatershed codes. 


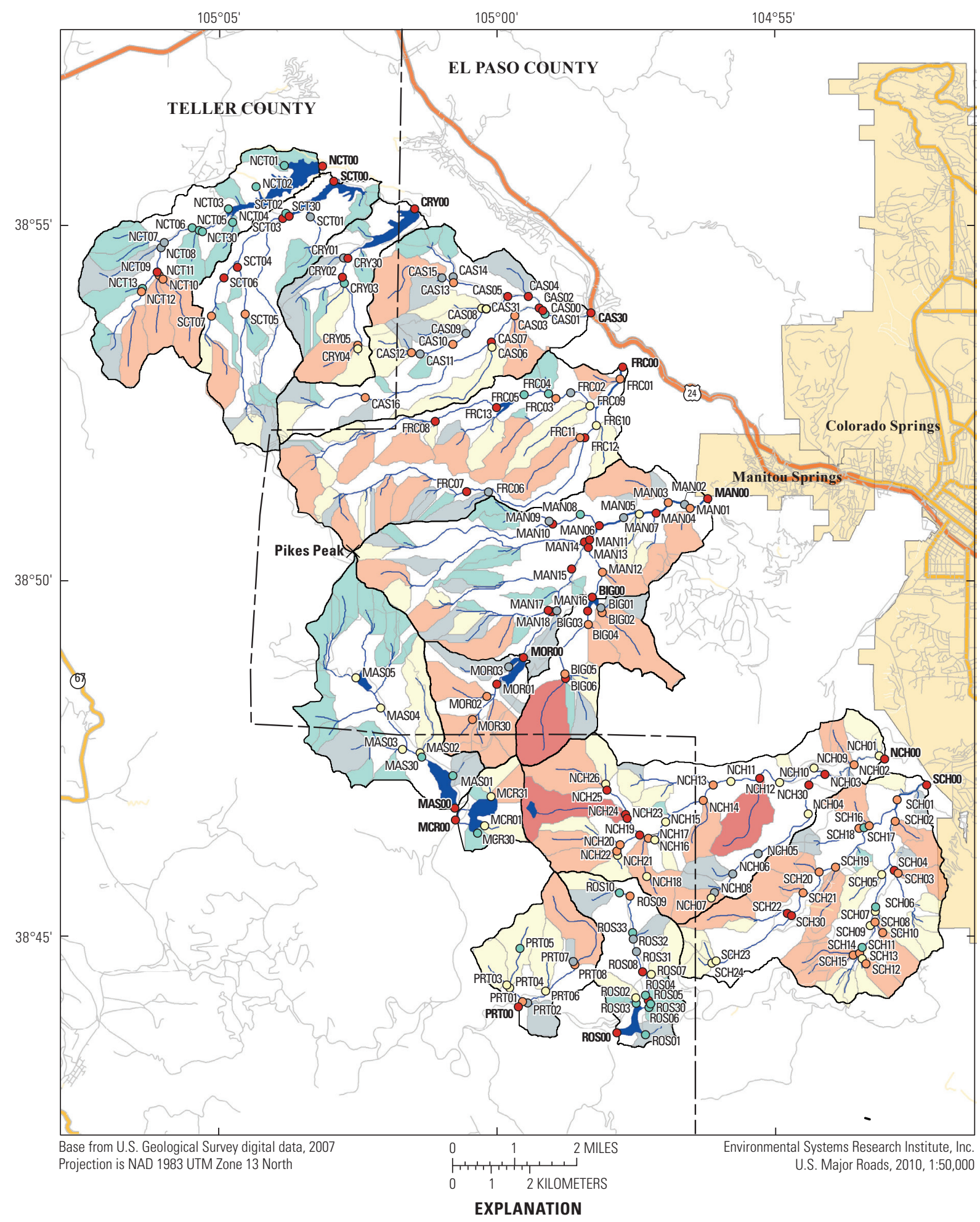

Estimated volume of debris flow at outlet of Strahler 3rd-order watershed (cubic meters)

\begin{tabular}{|c|}
\hline Less than 1,000 \\
\hline Greater than 1,000 to 2,000 \\
\hline Greater than 2,000 to 4,000 \\
\hline Greater than 4,000 to 10,000 \\
\hline Greater than 10,000 \\
\hline
\end{tabular}

MCR00 Primary watershed code

MCR30 Subwatershed code

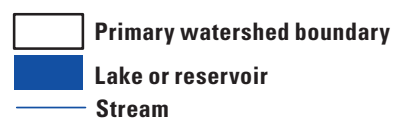

Stream
Estimated volume of debris flow at outlet of watershed of interest (cubic meters)

O Less than 1,000

O Greater than 1,000 to 2,000

O Greater than 2,000 to 4,000

- Greater than 4,000 to 10,000

Figure 9. Map of primary watersheds and subwatersheds showing estimated debris-flow volumes in response to the 25-year-recurrence, 1-hour-duration rainfall. See table 2 for watershed and subwatershed codes. 
In addition to the color-coded map symbols, the maps in figures 7, 8, and 9 include color-coded shaded areas representing the debris-flow volumes in Strahler third-order streams within the 170 subwatersheds. Although the contribution area upstream from the outlet of each Strahler third-order watershed is entirely shaded by a color representing the debris-flow estimated volume, the estimated volume is applicable only for a debris flow delivering sediment to the point at which smaller channels converge to form a Strahler third-order channel, and not for every channel segment upstream from that point. The shaded areas give a detailed breakdown of the debrisflow estimated volume in smaller areas of the subwatersheds, providing useful information for resource managers and emergency responders.

The debris-flow volume verification data presented in Cannon and others (2010, their figure 4) ranged between 100 and $100,000 \mathrm{~m}^{3}$ with predicted volumes within one order of magnitude of measured volumes. Using the precedent established by Cannon and others (2010), debris-flow volume estimates for the Pikes Peak study area are presented in table 2 as follows: (1) estimated volumes less than $100 \mathrm{~m}^{3}$ are reported as less than $100 \mathrm{~m}^{3}$, (2) estimated volumes greater than $100,000 \mathrm{~m}^{3}$ are reported as greater than $100,000 \mathrm{~m}^{3}$, (3) estimated volumes between $100 \mathrm{~m}^{3}$ and $1,000 \mathrm{~m}^{3}$ are rounded to the nearest hundred, and (4) estimated volumes between $1,000 \mathrm{~m}^{3}$ and $100,000 \mathrm{~m}^{3}$ are rounded to two significant digits.

The estimated volumes for potential postwildfire debris flows in the 170 subwatersheds in the Pikes Peak study area ranged from less than $100 \mathrm{~m}^{3}$ to greater than $100,000 \mathrm{~m}^{3}$ in response to the 2-year storm, the 10-year storm, and the 25 -year storm (table 2). Estimated debris-flow volumes for each subwatershed increased as the storm recurrence interval increased. Subwatersheds with the smallest estimated postwildfire debris-flow volumes tended to have small drainage areas, have a small percent area of steep hillslopes (table 2), and (or) be located in alpine and subalpine zones (figs. 7, 8, and 9). Subwatersheds with the largest estimated debris-flow volumes were those with the largest drainage areas. Forty-two subwatersheds had estimated debris-flow volumes equal to or greater than $10,000 \mathrm{~m}^{3}$ in response to a 25 -year storm and, of those, three had estimated debris-flow volumes greater than $100,000 \mathrm{~m}^{3}$ (table 2).

As with the probability estimates, debris-flow volume estimates for the 14 primary watersheds were evaluated separately because they consisted of nested subwatersheds (figs. 7-9). Postwildfire debris-flow volume estimates for the primary watersheds ranged from about 11,000 to greater than $100,000 \mathrm{~m}^{3}$ in response to the 2-year storm, from about 14,000 to greater than $100,000 \mathrm{~m}^{3}$ in response to the 10 -year storm, and from about 15,000 to greater than $100,000 \mathrm{~m}^{3}$ in response to the 25-year storm (table 1). The Cascade Creek (CAS), French Creek (FRC), Ruxton Creek (Manitou No. 1 Intake, $\mathrm{MAN}$ ), $\mathrm{NCH}$, and $\mathrm{SCH}$ primary watersheds each had estimated debris-flow volumes greater than $100,000 \mathrm{~m}^{3}$ in response to a 25-year storm (fig. 9 and table 2). These were the five largest primary watersheds, each having a watershed area greater than $20 \mathrm{~km}^{2}$.
Although some moderately to severely burned watersheds in the study area potentially can produce large volumes of debris-flow material (water, sediment, and other debris), determining where that material could be deposited below the watershed outlet is beyond the scope of this study. Wide and relatively low-gradient main-stem valleys in the primary watersheds, such as the lower reaches of Cascade Creek (CAS05, CAS13, CAS15) (fig. 9), potentially could intercept and capture some debris-flow material produced in tributaries before the material reaches the primary watershed outlet (CAS30) (fig. 9). The numerous reservoirs in the study area also would likely or almost certainly intercept debris-flow material from upstream areas before it could be transported to the primary watershed outlet.

\section{Combined Relative Debris-Flow Hazard Ranking}

The watersheds with the greatest potential postwildfire and postprecipitation debris-flow hazards are those with both high estimated probabilities of debris-flow occurrence and large estimated volumes of debris-flow material (Cannon and others, 2010). Results from the 25 -year storm debris-flow probability and volume equations were merged to produce a combined relative debris-flow hazard ranking for the 170 subwatersheds in the Pikes Peak study area to provide an overall indicator of the relative hazards associated with each subwatershed.

For each subwatershed, the debris-flow probability rank, with 1 associated with the highest probability (table 2), was added to the debris-flow volume rank, with 1 associated with the largest volume (table 2), to derive a preliminary combined rank sum. The preliminary combined rank sums for the 170 subwatersheds ranged from 22 (highest combined hazard) to 309 (lowest combined hazard). The preliminary combined rank sums for each subwatershed were renumbered with 1 assigned to the subwatershed with the highest combined hazard, 2 assigned to the subwatershed with the second-highest combined hazard, and so forth through 170 for the subwatershed with the lowest combined hazard.

The 10 subwatersheds with the highest combined relative debris-flow hazard rankings for the 25-year storm, listed generally from north to south, are

- CAS04 (rank 4) in the Cascade Creek watershed;

- BIG02 (rank 8) in the South Ruxton Creek watershed;

- MAN04 (rank 6) in the Ruxton Creek watershed;

- PRT01 (rank 9) in the Gould Creek watershed;

- ROS05 (rank 5) and ROS08 (rank 7) in the East Beaver Creek watershed;

- NCH11 (rank 3) and NCH19 (rank 10) in the North Cheyenne Creek watershed; and

- SCHO4 (rank 2) and SCH30 (rank 1) in the South Cheyenne Creek watershed (table 2). 
Combined relative debris-flow hazard rankings were not calculated for the 14 primary watersheds because the direct comparison of primary watershed rankings with the subwatershed rankings would be misleading. The primary watersheds were composite areas that generally consisted of the following: (1) multiple subwatersheds, for which individual debris-flow probabilities and volumes were estimated, and (2) interspersed, laterally planar hillslope areas, for which no individual debris-flow probability and volume estimates were made. Additionally, the outlets of several primary watersheds were located downstream from reservoirs.

\section{Summary and Conclusions}

Debris flows are fast-moving, high-density slurries of water, sediment, and debris that can have enormous destructive power. Debris flows typically are triggered by intense rainfall or rapid snowmelt on steep hillsides covered with erodible material. Although debris flows are a common geomorphic process in some unburned areas, a wildfire can transform conditions in a watershed with no recent history of debris flows into conditions that pose a substantial hazard to residents, communities, infrastructure, aquatic habitats, and water supply. In 2010, the U.S. Geological Survey, in cooperation with the City of Colorado Springs, Colorado Springs Utilities (CSU), initiated a prewildfire study to determine the potential for postwildfire debris flows in selected Pikes Peak area watersheds of El Paso and Teller Counties, Colo. The study objective was to estimate the probability of postwildfire debris flows and to estimate the approximate volumes of debris flows that could be delivered from 14 primary watersheds and 170 selected subwatersheds located within the primary watersheds with infrastructure of concern to CSU. This report presents the results of that study.

Debris-flow probabilities and volumes were estimated for 170 selected subwatersheds within the 14 primary watersheds in order to provide CSU with a relative measure of which subwatersheds might constitute the most serious debris-flow hazards in the event of a large-scale wildfire and subsequent rainfall. In addition to the outlets of these primary watersheds and subwatersheds, debris-flow probabilities and volumes at the outlets of Strahler third-order streams and their associated watersheds were estimated, providing useful information for resource managers and emergency responders. Presented graphically only, the shaded third-order watersheds give a visually detailed breakdown of the debris-flow probability and volume in smaller areas of the subwatersheds.

Using information provided in this report, CSU waterresource managers can plan prevention and mitigation strategies in advance of the occurrence of wildfires. Also, in the event of a large wildfire, this information will help managers identify the watersheds and subwatersheds with the greatest postwildfire debris-flow hazards. These estimates are hypothetical and neither the U.S. Geological Survey nor the United States Government may be held liable for any damages resulting from the authorized or unauthorized use of the estimates.
Potential postwildfire debris-flow probabilities and volumes in the study area were based on empirical equations. The 14 primary watersheds range in size from 3.20 to $42.65 \mathrm{~km}^{2}$ (1.24 to $16.47 \mathrm{mi}^{2}$ ), and the 170 subwatersheds range in size from 0.01 to $26.04 \mathrm{~km}^{2}\left(0.004\right.$ to $\left.10.06 \mathrm{mi}^{2}\right)$. The models assumed that all of the forest and shrub cover in the watershed would burn at moderate- to high-burn severity. Three postwildfire precipitation scenarios were used to represent a range of likely precipitation scenarios that could occur within 4 to 6 years after a wildfire: (1) a 2-year recurrence (50-percent annual exceedance probability), 1-hour-duration rainfall; (2) a 10-year recurrence (10-percent annual exceedance probability), 1-hourduration rainfall; and (3) a 25-year recurrence (4-percent annual exceedance probability), 1-hour-duration rainfall. Rainfall totals for Pikes Peak study area watersheds were determined from the National Oceanic and Atmospheric Administration and were considered to occur uniformly over each primary watershed.

The estimated probabilities for postwildfire debris flows in the 170 subwatersheds ranged from less than 1 to 46 percent in response to the 2-year storm (2-year recurrence, 1-hour duration rainfall), 1 to 67 percent in response to the 10 -year storm (10-year recurrence, 1-hour duration rainfall), and 1 to 72 percent in response to the 25-year storm (25-year recurrence, 1-hour duration rainfall). Postwildfire debris-flow probabilities for the 14 primary watersheds ranged from 4 to 42 percent in response to the 2-year storm, 8 to 64 percent in response to the 10 -year storm, and 10 to 70 percent in response to the 25-year storm.

Subwatersheds with the lowest postwildfire debris-flow probabilities tended to have large areas of alpine and subalpine vegetation or other large areas with sparse forest cover. Forty of the 170 subwatersheds had a greater than 60-percent probability of producing a debris flow in response to the 25-year storm. Subwatersheds with the highest probabilities tended to be heavily forested and tended to have a large percent area of steep slopes. Many of the subwatersheds with the highest debris-flow probabilities were tributaries in the Gould Creek (Platte Rogers Tunnel Intake, PRT), East Beaver Creek (Rosemont Reservoir, ROS), North Cheyenne Creek (NCH), and South Cheyenne Creek ( $\mathrm{SCH}$ ) primary watersheds.

The estimated volumes for potential postwildfire debris flows in the 170 subwatersheds in the Pikes Peak study area ranged from less than $100 \mathrm{~m}^{3}$ to greater than 100,000 $\mathrm{m}^{3}$ in response to the 2-year storm, the 10 -year storm, and the 25-year storm . Estimated debris-flow volumes for each subwatershed increased as the storm recurrence interval increased. Postwildfire debris-flow volume estimates for the 14 primary watersheds ranged from about 11,000 to greater than $100,000 \mathrm{~m}^{3}$ in response to the 2-year storm, from about 14,000 to greater than $100,000 \mathrm{~m}^{3}$ in response to the 10 -year storm, and from about 15,000 to greater than $100,000 \mathrm{~m}^{3}$ in response to the 25 -year storm. Subwatersheds with the smallest estimated postwildfire debris flow volumes tended to have small drainage areas, have a small percent area of steep hillslopes, and (or) be located in alpine and subalpine zones. Subwatersheds with the largest estimated debris-flow volumes were those with the largest drainage areas. 
The watersheds with the greatest potential postwildfire and post-precipitation hazards are those with both high estimated probabilities of debris-flow occurrence and large estimated volumes of debris-flow material. The 10 subwatersheds with the greatest combined relative debris-flow hazard rankings for the 25-year storm are CAS04 in the Cascade Creek watershed, BIG02 in the South Ruxton Creek watershed, MAN04 in the Ruxton Creek watershed, PRT01 in the Gould Creek watershed, ROS05 and ROS08 in the East Beaver Creek watershed, NCH11 and NCH19 in the North Cheyenne Creek watershed, and $\mathrm{SCHO} 4$ and $\mathrm{SCH} 30$ in the South Cheyenne Creek watershed.

Although the location, percentage of burned area, severity of wildfire, and subsequent storm intensity and duration cannot be known in advance, hypothetical scenarios, such as those used in this report, are useful planning tools for conceptualizing potential postwildfire debris-flow hazards. The models in this study were used only to estimate postwildfire debris-flow characteristics at a specific location: the watershed outlet. No attempt was made in this study to model the transport of debris-flow material downstream from the watershed outlet. Substantial flooding and other fluvial-erosion processes that could cause substantial damage also can occur under postwildfire conditions, but were beyond the scope of this study.

\section{Acknowledgments}

This study was performed by the U.S. Geological Survey in cooperation with the City of Colorado Springs, Colo., Colorado Springs Utilities. Earl Cassidy and Katherine Chase of the USGS, performed technical reviews of the report. Photographs were taken by John G. Elliott and Keelin R. Schaffrath, USGS.

\section{References Cited}

Cannon, S.H., 2001, Debris-flow generation from recently burned drainage basins: Environmental and Engineering Geoscience, v. 7 no. 4, p. 321-341.

Cannon, S.H., Powers, P.S., and Savage, W.Z., 1998, Firerelated hyperconcentrated and debris flows on Storm King Mountain, Glenwood Springs, Colorado, USA: Environmental Geology, v. 34, no. 2-3, p. 210-218.

Cannon, S.H., Gartner, J.E., Rupert, M.G., Michael, J.A., Rea, A.H., and Parrett, C., 2010, Predicting the probability and volume of postwildfire debris flows in the intermountain western United States: Geological Society of America Bulletin, v. 122, p. 127-144.

Costa, J.E., 1988, Rheologic, geomorphic, and sedimentologic differentiation of water floods, hyperconcentrated flows, and debris flows, in Flood geomorphology: New York, John Wiley and Sons, p. 113-122.
Costa, J.E., and Jarrett, R.D., 1981, Debris flows in small mountain stream channels of Colorado and their hydrologic impacts: Bulletin of the Association of Engineering Geologists, v. XVIII, no. 3, p. 309-322.

Das, B.M., 1983, Advanced soil mechanics: New York, McGraw-Hill, $511 \mathrm{p}$.

Elliott, J.G., Flynn, J.L., Bossong, C.R., and Char, S.J., 2011, Estimated probabilities and volumes of postwildfire debris flows, a prewildfire evaluation for the Upper Blue River watershed, Summit County, Colorado: U.S. Geological Survey Scientific Investigations Report 2006-5039, 22 p. http://pubs.usgs.gov/sir/2011/5039/

Elliott, J.G., Smith, M.E., Friedel, M.J., Stevens, M.R., Bossong, C.R., Litke, D.W., Parker, R.S., Costello, Catherine, Wagner, Jason, Char, S.J., Bauer, M.A., and Wilds, S.R., 2005, Analysis and mapping of post-wildfire hydrologic hazards for the 2002 Hayman, Coal Seam, and Missionary Ridge wildfires, Colorado: U.S. Geological Survey Scientific Investigations Report 2004-5300, 104 p.

Environmental Systems Research Institute, Inc., 2009, ArcGIS Desktop (v. 9.3.1, Service Pack 1): Redlands, Calif.

Gartner, J.E., Cannon, S. H., Bigio, E.R., Davis, N.K., Parrett, C., Pierce, K.L., Rupert, M.R., Thurston, B.L., Trebish, M.J., Garcia, S.P., and Rea, A.H., 2005, Compilation of data relating to the erosive response of 606 recently burned basins in the Western U.S.: U.S. Geological Survey OpenFile Report 05-1218, http://pubs.usgs.gov/of/2005/1218.

Gartner, J.E., Cannon, S. H., Santi, P.M., and Dewolfe, V.G., 2008, Empirical models to predict the volumes of debris flows generated by recently burned basins in the western U.S.: Geomorphology, v. 96, p. 339-354, doi: 10.1016/j.geomorph.2007.02.033.

Gesch, D., Oimoen, M., Greenlee, S., Nelson, C., Steuck, M., and Tyler, D., 2002, The national elevation dataset: Photogrammetric Engineering and Remote Sensing, v. 68, no. 1, p. 5-11.

Green, G.N., 1992, The Digital Geologic Map of Colorado in ARC/INFO Format: U.S. Geological Survey OpenFile Report 92-0507, 9 p.; http://pubs.usgs.gov/of/1992/ ofr-92-0507

Griffiths, P.G., Webb. R.H., and Melis, T.S., 1996, Initiation and frequency of debris flows in Grand Canyon, Arizona: U.S. Geological Survey Open-File Report 96-491, 35 p.

Helsel, D.R., and Hirsch, R.M., 2002, Statistical methods in water resources: U.S. Geological Survey Techniques of Water-Resources Investigations, book 4, chap. A3, 510 p., http://pubs.usgs.gov/twri/twri4a3/html/pdf_new.html (accessed February 26, 2010).

Horton, R.E., 1945, Erosional developments of streams and their drainage basins; hydrophysical approach to quantitative morphology: Geol. Soc. Am. Bull., v. 56, p. 275-370. 
Hosmer, D.W., and Lemeshow, Stanley, 2000, Applied logistic regression, 2d ed.: New York, John Wiley \& Sons, Inc., 375 p.

Hungr, O., Morgan, G.C., and Kellerhals, R., 1984, Quantitative analysis of debris-torrent hazards for design of remedial measures: Canadian Geotechnical Journal, v. 21, p. 663-677.

Keefer, D.K., and Johnson, A.M., 1983, Earth flowsMorphology, mobilization, and movement: U.S. Geological Survey Professional Paper 1264, 56 p.

Kuhn, Gerhard, 2005, Historical perspective of statewide streamflows during the 2002 and 1977 droughts in Colorado: U.S. Geological Survey Scientific Investigations Report 2005-5174, 84 p.

Lindsey, Rebecca, 2002, Satellites aid burn area rehabilitation: National Aeronautic and Space Administration online report, various pagination, available online at http://earthobservatory.nasa.gov/Study/BAER/ (accessed February 15, 2007).

Melton, M.A., 1965, The geomorphic and paleoclimate significance of alluvial deposits in southern Arizona: Journal of Geology, v. 73, p. 1-38.

Miller, J.F., Frederick, R.H., and Tracey, R.J., 1973, Precipitation-frequency atlas of the western United States, v. 3 Colorado: Silver Spring, Md., U.S. Department of Commerce, National Oceanic and Atmospheric Administration, National Weather Service.

Nakagaki, N., Price, C.V., Falcone, J.A., Hitt, K.J., and Ruddy, B.C., 2007, Enhanced National Land Cover 1992 (NLCDe 92), U.S. Geological Survey Sacramento, CA [online-only digital data set] http://water.usgs.gov/lookup/ getspatial?nlcde92.

Pielke, R.A., Sr., Doeskin, N., Bliss, O., Green, T., Chaffin, C., Salas, J.D., Woodhouse, C.A., Lukas, J., and Wolter, K., 2005, Drought 2002 in Colorado-An unprecedented drought or a routine drought?: Pure and Applied Geophysics, v. 162, p. 1455-1479.

Pierson, T.P., 2005, Distinguishing between debris flows and floods from field evidence in small watersheds: U.S. Geological Survey Fact Sheet 2004-3142, 4 p.
Pierson, T.C., and Costa, J.E., 1987, A rheological classification of subaerial sediment-water flows: Geological Society of America, Reviews in Engineering Geology, v. VII, 12 p.

Ruddy, B.C., Stevens, M.R., Verdin, K.L., and Elliott, J.G., 2010, Probability and volume of potential postwildfire debris flows in the 2010 Fourmile burn area, Boulder County, Colorado: U.S. Geological Survey Open-File Report 2010-1244, 5 p.

Schwartz, G.E., and Alexander, R.B., 1995, State Soil Geographic (STATSGO) data base for the conterminous United States: U.S. Geological Survey Open-File Report 95-449.

SPSS, Inc., 2000, SYSTAT 10, Statistics I-Software documentation: Chicago, SPSS, Inc., 663 p.

Strahler, A.N., 1957, Quantitative analysis of watershed geomorphology: American Geophysical Union Transactions, v. 38, p. 913-920.

Stevens, M.R., Bossong, C.R., Litke, D.W., Viger, R.J., Rupert, M.G., and Char, S.J., 2008, Estimated probability of post-wildfire debris-flow occurrence and estimated volume of debris flows from a pre-wildfire analysis in the Three Lakes Watershed, Grand County, Colorado: U.S. Geological Survey Scientific Investigations Map 3009, 1 sheet.

U.S. Department of Agriculture, National Resources Conservation Service, National Soil Survey Center, 1991, State Soil Geographic (STATSGO) database: Data use information, Miscellaneous Publication Number 1492, 110 p. (Revised July 1994.)

U.S. Geological Survey, 2010, Streamstats-A water resources web application, accessed July 19, 2010, http://water.usgs.gov/ osw/streamstats/colorado.html.

Verdin, K.L. and Greenlee, S., 2003, Continuous parameterization using EDNA, in Proceedings of the 2003 ESRI User's Conference, July 7-11, 2003, San Diego, California. URL: http://gis.esri.com/library/userconf/proc03/p0617.pdf, accessed May 2007.

Verdin, K. L. and Worstell, B., 2008, A fully distributed implementation of mean annual streamflow regional regression equations: Journal of the American Water Resources Association, v. 44, p. 1537-1547. doi: 10.1111/ j.1752-1688.2008.00258.x

Publishing support provided by:

Denver Publishing Service Center, Denver, Colorado

For more information concerning this publication, contact:

Director, USGS Colorado Water Science Center

Box 25046, Mail Stop 415

Denver, C0 80225

(303) 236-4882

Or visit the Colorado Water Science Center Web site at: http://co.water.usgs.gov/

This report is available at: http://pubs.usgs.gov/sir/2012/5104/ 


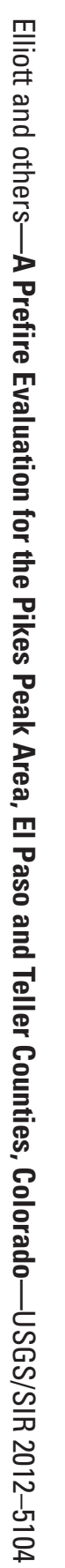

